\title{
Comparison of Methods to Predict Lower Bound Buckling Loads of Cylinders under Axial Compression
}

\author{
Waddy T. Haynie* and Mark W. Hilburger ${ }^{\dagger}$ \\ NASA Langley Research Center, Hampton, VA, 23681-2199, USA
}

\begin{abstract}
Results from a numerical study of the buckling response of two different orthogrid stiffened circular cylindrical shells with initial imperfections and subjected to axial compression are used to compare three different lower bound buckling load prediction techniques. These lower bound prediction techniques assume different imperfection types and include an imperfection based on a mode shape from an eigenvalue analysis, an imperfection caused by a lateral perturbation load, and an imperfection in the shape of a single stress-free dimple. The STAGS finite element code is used for the analyses. Responses of the cylinders for ranges of imperfection amplitudes are considered, and the effect of each imperfection is compared to the response of a geometrically perfect cylinder. Similar behavior was observed for shells that include a lateral perturbation load and a single dimple imperfection, and the results indicate that the predicted lower bounds are much less conservative than the corresponding results for the cylinders with the mode shape imperfection considered herein. In addition, the lateral perturbation technique and the single dimple imperfection produce response characteristics that are physically meaningful and can be validated via testing.
\end{abstract}

\section{Introduction}

I IGHT-WEIGHT, thin-walled, circular cylindrical shells, used in aerospace applications, are inherently Lstability-critical structures. For circular cylinders subjected to axial compression, linear buckling analyses predict un-conservative buckling, or critical, loads when compared to corresponding experimental results. This un-conservative prediction and the corresponding uncertainty in the actual buckling load of the as-built structure can lead to significant challenges for the designer of these structures. The traditional approach for designing a thin-walled, buckling-resistant shell structure is to predict the buckling load of the shell with a deterministic, linear bifurcation buckling analysis, and then to reduce this predicted load with an empirical 'knockdown' factor (e.g. Ref. 1). The analysis is usually based on nominal structural dimensions and material properties of an idealized, geometrically perfect shell. The empirical knockdown factor is intended to account for the difference between the predicted buckling load and the actual buckling load for the shell determined from tests. For example, the design knockdown factor used in the design of buckling-resistant cylindrical shells is often based on the 'lower-bound' design recommendations reported in NASA SP $8007 .{ }^{1}$ This design philosophy has been shown to result in overly conservative designs for these structures, but it can potentially even result in un-conservative designs if the empirical data are not representative of the design of interest.

While it is generally recognized that initial geometric shell-wall imperfections are a major contributor to the discrepancy between the predicted shell buckling loads and the experimentally measured shell buckling loads (e.g., Refs. 2-6), the traditional sources of design knockdown factors do not include data or information related to the sensitivity of the response of a shell to various forms of imperfections. In addition, the traditional sources of design knockdown factors for predicting shell buckling loads do not include information for shell structures made from state-of-the-art materials and manufacturing processes. Recent studies (e.g., Refs. 7-11) have shown that traditional initial geometric shell-wall imperfections, and other nontraditional

*Aerospace Engineer, Structural Mechanics and Concepts Branch, 8 W. Taylor St., Mail Stop 190. Member, AIAA
†Senior Research Engineer, Structural Mechanics and Concepts Branch, 8 W. Taylor St., Mail Stop 190. Senior Member, AIAA 
forms of imperfections or variations in geometric and material parameters, loading conditions, and boundary conditions can significantly affect the buckling load of a compression-loaded composite shell structure. Other recent studies (e.g., Ref. 12) have identified the effects of fabrication related anomalies on the buckling loads of metallic shells.

Furthermore, Starnes et al. ${ }^{10}$ and Hilburger and Starnes ${ }^{11}$ have shown that when structural details such as as-measured shell-wall geometry, shell wall thickness, and non-uniform loading are included in a highfidelity analysis, the buckling load and transient dynamic collapse behavior can be predicted accurately. However, these structural details are typically not known a priori and, thus, designers have had to resort to other methods in order to predict conservative values of the critical load for design. In particular, analysisbased lower bound buckling load predictions can be used and three such methods are presented herein. The first is a common approach in which a mode shape corresponding to the first eigenvalue from a buckling analysis, or eigenmode, is used as an initial geometric imperfection shape. Using this approach, a lower bound prediction for the buckling load can be determined by increasing the amplitude of the imperfection to an amplitude larger than the amplitude of any imperfection that results from manufacturing. However, the eigenmode shape is typically not representative of a true as-built imperfection shape. Furthermore, an eigenmode imperfection is not always the most detrimental imperfection shape. The second method is a relatively new approach developed by Hühne et al. ${ }^{13}$ that uses a lateral perturbation load to create a local dimple-shaped imperfection in the shell wall. This dimple is typically similar to the type of dimple that forms in a compression-loaded shell at the onset of buckling as observed in tests and is thus a physically meaningful initial imperfection or perturbation. In this analysis-based lower-bound approach, the perturbation load is applied before the axial load is applied and is held constant during the application of the axial load. Hühne et al. determined that, for unstiffened composite cylinders, there exists a range of lateral perturbation loads where the shell buckling load is very sensitive to changes in the magnitude of the perturbation load. However, above a certain threshold value of the perturbation load, the buckling load appears to reach a minimum value or lower bound and is not sensitive to variations in the perturbation load. Hühne et al. consider the buckling load at the threshold value to be a reasonable approximation of a lower bound to the buckling load of the shell. The third method considered herein uses the deformed shape resulting from applying the lateral perturbation load to a cylinder before any axial load is applied. This imperfection shape provides a stress-free analogue to the lateral perturbation load imperfection, for which local stresses exist near the point of application of the perturbation load.

In this study, numerically predicted responses of two different geometrically perfect and imperfect orthogridstiffened circular cylinders subjected to axial compressive loads are used to compare several lower bound buckling load prediction methods. The three types of initial imperfections described above are used for predicting lower bound buckling loads. These imperfections will be referred to as the mode shape imperfection, lateral perturbation load imperfection, and single dimple imperfection. The response of a geometrically perfect cylinder is also determined to serve as a baseline to which the imperfect cylinders can be compared.

\section{Problem Description}

\section{A. Geometry, Loading, and Boundary Conditions}

The geometry of the cylinders examined in this study is described in Fig. 1. The surface coordinate system $(x, s, z)$ is defined with its origin at one end of the cylinder. The axial coordinate $x$ and the circumferential arc length coordinate $s$ describe the cylinder surface, and the $z$ coordinate describes the direction normal to the surface. The cylinder has a length $L$, circumference $C$, radius $R$, and skin thickness $h$. The cylinders have internal integral blade stiffeners in the axial and circumferential directions, referred to as an orthogrid pattern. In Fig. 1 a portion of the cylinder outer skin is removed to show the stiffener layout. The spacing of the circumferential stiffeners, or ring stiffeners, is $b_{r}$, and the spacing of the axial stiffeners, or stringers, is $b_{s}$. The thicknesses of the ring stiffeners and stringers are $t_{r}$ and $t_{s}$, respectively. The heights of the ring stiffeners and stringers, $h_{r}$ and $h_{s}$, respectively, are measured from the inner skin surface. The total wall thickness $H$ is the sum of the cylinder wall thickness, $h$, and either the ring stiffener or stringer height, $h_{r}$ or $h_{s}$, and since within this study $h_{r}=h_{s}$, there is only one value of $H$ for a given cylinder.

Two cylinders, referred to as full-scale and sub-scale cylinders, are studied and the specific geometries are given in Table 1. The geometry of the full-scale cylinder is chosen because it is of a similar size to current launch vehicle designs. However, due to the costs associated with testing a full-scale structure, it is often more practical to first consider the response of smaller laboratory-scale structures that contain similar 


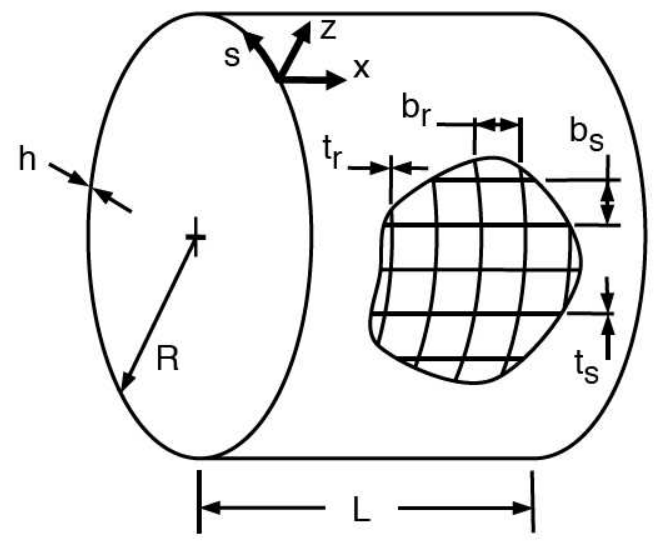

Figure 1. Cylinder geometry and coordinate system

design features to large-scale launch-vehicle-type structures. As such, the sub-scale cylinder, at a scale more feasible for laboratory testing, is also considered. It should be noted that the sub-scale cylinder cannot be scaled exactly from the full-scale cylinder because of manufacturing limitations on material thicknesses and machining dimensions.

The boundary conditions and loading are described in Fig. 2. Both ends of the cylinder are clamped with $w=v=$ $\partial w / \partial x=0$. At $x=0$ an axial end displacement $u=\delta$ is applied, while the other end, at $x=L$, is fixed $(u=0)$. The axial load $P$, not shown in the figure, is measured at $x=0$, and is the net load on the end of the cylinder. The axial load is positive for a compressive load and negative for a tensile load. For the cases when a lateral perturbation load, $Q$, is applied, it is applied at the cylinder mid-length $x=L / 2$, normal to the surface, and toward the inside of the cylinder.

\section{B. Material Properties}

The cylinders in this study are assumed to be fabricated of an Aluminum-Lithium (Al-Li) alloy, which is a typical material for space launch vehicles. Although some Al-Li alloys exhibit anisotropic behavior, especially when considering the strengths in different material directions, the material in this study is considered to be isotropic and linear elastic. The material stiffness is $E=10.6 \times 10^{6} \mathrm{psi}$, and Poisson's ratio is 0.33 .

Table 1. Cylinder and stiffener geometry*

\begin{tabular}{|l|c|c|}
\hline & full-scale & sub-scale \\
\hline Cylinder \\
\hline length, $L$ & 144 & 72 \\
\hline radius, $R$ & 108 & 48 \\
\hline skin thickness, $h$ & 0.14 & 0.10 \\
\hline Axial Stiffener \\
\hline spacing, $b_{s}$ & 5.5 & 3.0 \\
\hline height, $h_{s}$ & 0.50 & 0.125 \\
\hline width, $t_{s}$ & 0.36 & 0.40 \\
\hline Circumferential Stiffener \\
\hline spacing, $b_{r}$ & 5.1 & 3.0 \\
\hline height, $h_{r}$ & 0.50 & 0.125 \\
\hline width, $t_{r}$ & 0.36 & 0.40 \\
\hline
\end{tabular}

*all dimensions are in inches

\section{Finite Element Model}

The finite element analyses are performed using the STAGS finite element code. ${ }^{14}$ A representative mesh of the finite-element model for both cylinder geometries is shown in Fig. 2. The model is comprised of four-noded quadrilateral 410 shell elements. This element is formulated using the Kirchhoff hypothesis and a nonlinear Lagrangian strain tensor. There are six degrees of freedom at each node: three translational and three rotational. One of the rotational degrees of freedom is the drilling degree of freedom, which corresponds to rotation about the normal to the surface of the element. The element meshes for both cylinders have a uniform element length in the circumferential direction. Two element lengths are used in the axial direction. A shorter axial element length is used near the ends of the cylinder to accurately model the bending response near the end of the shell, and a longer length is used in the middle of the cylinder where 
the bending response has attenuated. The stiffeners are incorporated in the model using a smeared stiffener approach. The clamped boundary condition at $x=L$ is enforced by fixing all degrees of freedom. At $x=0$ the boundary condition is enforced by fixing all degrees of freedom except for the the axial component. The cylinder is loaded by applying an axial displacement $u=\delta$ at $x=0$, and a constraint is prescribed such that all nodes on this end are displaced an equal amount. The force $P$ required to produce a given end displacement is the sum of the forces on the nodes at this end of the cylinder.

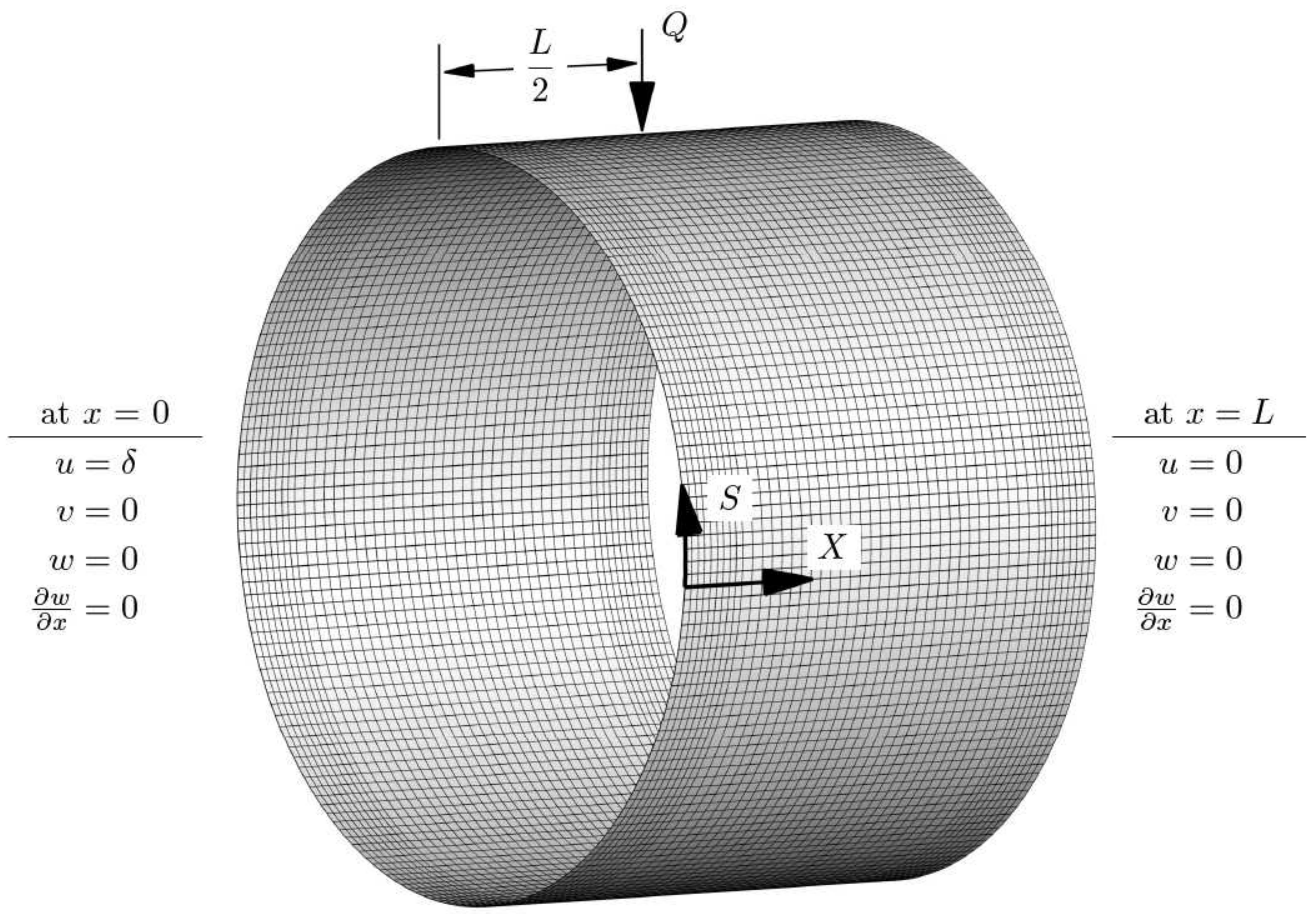

Figure 2. Boundary conditions, loading, and representation of finite-element mesh

\section{Numerically-Predicted Cylinder Response}

Four cases for the full-scale and sub-scale cylinders under an axial compressive load were considered; a perfect geometry, to serve as a baseline, and three types of imperfections. A lower-bound buckling load was determined for each of the three imperfections. The responses of the perfect cylinders are discussed in the following section. Then the responses of the imperfect cylinders are presented in the subsequent sections and include cylinders with an imperfection based on the mode shape from a linear bifurcation buckling analysis, an imperfection caused by a lateral perturbation load, and a single stress-free dimple imperfection. In the last section, the lower bound buckling loads for each imperfection type are compared.

\section{A. Response of a Perfect Cylinder}

The response of a geometrically perfect cylinder was determined using STAGS and will serve as a baseline to compare the effects of the different imperfections. The prebuckling, buckling, and postbuckling responses were determined for the full-scale and sub-scale cylinders. The approach used to determine the response of the perfect cylinders presented herein was also followed for the imperfect cylinders.

The prebuckling response was determined by using the Riks' arc length path-following method, ${ }^{15}$ but with a modification made to the solution step control that enabled STAGS to converge to a critical value (limit point) without requiring several manual restarts. The standard path-following routine in STAGS was modified so that any solution step that was unstable (contained negative roots in the tangent stiffness matrix) was not accepted as a converged step. The solution process was then automatically restarted from the prior 
stable solution and the step size was reduced by half. This sequence would continue until a stable equilibrium solution was obtained. However, if no additional stable solutions were obtained after a predefined number of solution step cuts and restarts occurred, then the analysis would stop. The critical axial end displacement $\delta^{c r, p e r}$ for the perfect cylinders was determined using this subroutine, and the critical axial load $P^{c r, p e r}$ was the load calculated by STAGS at the critical axial end displacement.

Once a critical point was obtained, the buckling response was predicted by using a nonlinear transient analysis. The buckling response was initiated in the transient analysis by slightly increasing the applied end displacement of the cylinder from the critical value to a value where the cylinder is in an unstable configuration. This small increase in end displacement was no more than $0.2 \%$ of the critical value. During the transient analysis, the end displacement was held constant and a variable time step was used in an implicit time integration scheme. Rayleigh's proportional damping was used to damp out the kinetic energy and establish a stable postbuckling configuration in the cylinder. Once the cylinder settled into a stable configuration, which was indicated by the kinetic energy damping out to a very small value, the transient analysis was stopped, and a nonlinear static analysis was restarted with the shell in the new postbuckling configuration to verify it was in a stable state.

After establishing a stable initial postbuckling state, the postbuckling response of the cylinders was determined using nonlinear static analyses. For the first few solution steps in the postbuckling region, a simple displacement control algorithm was used because multiple solution paths were present near the initial postbuckling solution and it was observed that the Riks' arc length method had difficulty finding a single path to follow. After obtaining several solutions with the displacement control algorithm, the analysis was transitioned back to using the Riks' arc length method. As in the prebuckling region, the stable pathfollowing subroutine was used where unstable solutions were not accepted. This approach was used to determine the response deep into the postbuckling range.

Using the procedure described above, the responses of the full-scale and sub-scale perfect cylinders were determined, and are shown in Figs. 3 and 4, respectively. The load vs. axial end displacement responses are shown in Figs. 3a and 4a. The results are normalized by the critical values of load and end displacement determined from the prebuckling analyses. For the full-scale cylinder, the critical axial end displacement was $\delta_{\text {full }}^{c r, p e r}=0.20$ inches, and the critical axial load was $P_{\text {full }}^{\text {cr,per }}=1.78 \times 10^{6} \mathrm{lbs}$. For the sub-scale cylinder, the critical values were $\delta_{\text {sub }}^{c r, p e r}=0.188$ inches and $P_{\text {sub }}^{c r, p e r}=9.9 \times 10^{5} \mathrm{lbs}$. The cylinder axial stiffness, the slope of the load vs. end displacement response, was $8.8 \times 10^{6} \mathrm{lb} /$ in and $5.3 \times 10^{6} \mathrm{lb} /$ in for the full-scale and sub-scale cylinders, respectively. In Figs. 3a and $4 \mathrm{a}$ three points are used to describe the response as the cylinder moves from a prebuckling state to an initial postbuckling state. The letters identifying these points refer to the subfigure in which the configuration corresponding to the point appears. For each of these points, the cylinder shape is shown along with a color contour plot of the normalized normal displacements $w / H$ on a 'rolled out' view of the cylinder surface. The first point (c), marked by the diamond symbol, is the critical or buckling point and corresponds to the axisymmetric displacement response shown in Figs. 3c and 4c. When the cylinder buckles, the configuration first changes to a short wavelength buckling pattern, Figs. $3 \mathrm{~d}$ and $4 \mathrm{~d}$. This change in configuration corresponds to a significant reduction in axial load in the cylinder, marked by (d) and the square symbol in Figs. 3a and 4a, and is referred to as the intermediate buckling configuration. Point (d) is also marked in the load history plot, shown in Figs. $3 \mathrm{~b}$ and $4 \mathrm{~b}$, where it can be seen that the cylinder momentarily settles into this configuration before transitioning into the initial postbuckling configuration, point (e). The intermediate configuration of the full-scale cylinder has sixteen circumferential waves and five axial half waves, and the sub-scale cylinder has twelve circumferential waves and four axial half waves. Though not shown, the full-scale cylinder was observed to briefly have configurations with seven then six axial half waves before settling into the intermediate configuration with five axial half waves. Similarly, the sub-scale cylinder briefly developed into a configuration with five axial half waves before settling into the intermediate configuration with four axial half waves. The transition from the intermediate buckling configuration to the initial stable postbuckling configuration was characterized by a coalescence of buckles, that is, a reduction in the number of circumferential and axial waves. In the initial postbuckling configuration, both cylinders exhibited two axial half waves, and the full-scale and sub-scale cylinders had eleven and nine circumferential waves, shown in Figs. 3e and 4e, respectively. The reduction in load from the prebuckling configuration to the initial postbuckling configuration is greater than $50 \%$ from the critical load for both cylinders. Having described the response of the perfect cylinders, the effect of the imperfections on the cylinder response will be discussed next. 


\section{B. Response of a Cylinder with a Mode Shape Imperfection}

The mode shapes used as an initial geometric imperfection were calculated by STAGS eigenvalue analyses and assumed a linear stress state. Only the mode shape for the first eigenvalue was considered as an imperfection shape for each of the shells considered herein. It is understood that the mode shape for the first eigenvalue might not always be the most detrimental imperfection shape, but it was deemed sufficient for the purpose of illustration in this study. More specifically, it was necessary to have an imperfection shape that would be representative of this method to predict a lower bound buckling load, and it was assumed that this choice for the mode shape imperfection satisfied that criterion. The mode shapes for the full-scale and sub-scale cylinders are shown in Fig. 5, with the deformed shape shown on the left and a color contour plot of the normal displacement of the cylinder surface plotted on the 'rolled-out' surface on the right. For the full-scale cylinder, Fig. 5a, there were sixteen circumferential full waves and seven axial half waves in the mode shape. Each wavelength in the circumferential direction spanned the equivalent of 7.8 longitudinal stiffeners. For the sub-scale cylinder, Fig. 5b, there were twelve circumferential full waves and five axial half waves in the mode shape. The wavelength in the circumferential direction spanned the equivalent of 8.4 longitudinal stiffeners. The displacements in each mode shape were normalized so the largest magnitude had a value of unity. Using the imperfection shapes for the respective cylinders, imperfection amplitudes over the range of $0.010 \leq a / H \leq 1.0$ were considered for both the full-scale and sub-scale cylinders. This range was assumed to be sufficient to include a practical range of imperfection amplitudes that would be acceptable for as-built cylinders.

The critical, or limit, load $P^{c r}$ of the cylinders for each imperfection amplitude was determined using nonlinear static analyses. The same stable path following subroutine that was used for the perfect cylinder, described in the previous section, was used for these analyses as well. The results of the analyses are summarized in Figs. 6 and 7 for the full-scale and sub-scale cylinders, respectively. The critical loads for each of the imperfection amplitudes considered are shown in Figs. 6a and 7a, and in the corresponding load vs. end displacement responses shown in Figs. $6 \mathrm{~b}$ and $7 \mathrm{~b}$ each terminates at the critical load. The load vs. axial displacement responses for the bounding values of the imperfection amplitude $a / H=0.010$ and 1.00 are plotted in red. Responses for two selected intermediate imperfection amplitudes are plotted in blue, and the significance of these intermediate results will be discussed below. Also, the corresponding responses of the perfect cylinders which include the postbuckling response and the square symbol, shown in Figs. 3a and $4 \mathrm{a}$, are repeated in Figs. $6 \mathrm{~b}$ and $7 \mathrm{~b}$.

Initially, increasing the imperfection amplitude decreased the critical load of the cylinder as expected. At an imperfection amplitude of $a / H=0.20$ for the full-scale cylinder and $a / H=0.140$ for the sub-scale cylinder, a local minimum for $P^{c r}$ occurred. The load vs. end displacement responses for these imperfections are indicated by blue lines in Figs. $6 \mathrm{~b}$ and $7 \mathrm{~b}$, and the critical loads are marked by blue triangles. The values of the critical load at these imperfection amplitudes were $P^{c r}=0.63 P_{f u l l}^{c r, p e r}$ and $P^{c r}=0.66 P_{\text {sub }}^{c r, p e r}$ for the full-scale and sub-scale cylinders, respectively. When the imperfection amplitudes were increased beyond these values, $P^{c r}$ increased. At values of $a / H=0.65$ and $a / H=0.45$ for the full-scale and sub-scale cylinders, respectively, a local maximum is reached in the $P^{c r}$ vs. imperfection amplitude curve. The peak loads were $P^{c r}=0.72 P_{\text {full }}^{c r, p e r}$ and $P^{c r}=0.78 P_{\text {sub }}^{c r, p e r}$ for the full-scale and sub-scale cylinders, respectively. It is noted that a curve traced by the critical loads for the various imperfection amplitudes in Figs. 6b and $7 \mathrm{~b}$, would be the same as, or fall under, a postbuckling equilibrium path predicted for the cylinder's respective mode shape using an analytical approach or allowing unstable solution steps within a finite element analysis. For imperfection amplitudes greater than those at which the local maximum load occurred, the end displacement at which the maximum load occurred also decreased. This indicates either a sharp change in the postbuckling equilibrium path or that another postbuckling equilibrium path is determining the path traced by the maximum loads.

When using a mode shape imperfection, especially ones with relatively short wavelengths like in the mode shapes used considered in this study, a significant reduction in the axial stiffness of the cylinder occurs with increases in the imperfection amplitude. This behavior can be seen in Figs. $6 \mathrm{~b}$ and $7 \mathrm{~b}$, by the reduction in slope of the load vs. end displacement curves and is quantified in Fig. 8 for the full-scale and sub-scale cylinders. The axial stiffness was normalized by the axial stiffness of the perfect cylinder. To determine the axial stiffness of a cylinder for a given imperfection amplitude, only the range $0<\delta<0.5 \delta^{c r, p e r}$ of the load vs. end displacement curve was used. The slope was determined by performing a linear regression on the data, and in all cases the $R^{2}$ value, a measure of the fit of each linear regression, was greater than 0.99 . Over the range of imperfection amplitudes considered, the axial stiffness was reduced by $40 \%$ for both the full- 
scale and sub-scale cylinders. For end displacements greater than $\delta>0.5 \delta^{c r}$, the load vs. end displacements become nonlinear and the reduction in the axial stiffness becomes significantly greater for increasing end displacement. This effect is more pronounced for larger values of the imperfection amplitude.

Based on the effect of the imperfection amplitude on the maximum loads for the cylinders, shown in Figs. 6a and 7a, lower bounds for the buckling load are predicted to be $P^{c r}=0.63 P_{\text {full }}^{c r, p e r}$ and $P^{c r}=$ $0.66 P_{s u b}^{c r, p e r}$ for the full-scale and sub-scale cylinders, respectively. These values are taken from the local minimums that occur at $a=0.20 H$ and $a=0.14 H$ for the full-scale and sub-scale cylinders, respectively. The lower bounds were established using these points instead of the actual minimums that occurred at $a=H$ because imperfection amplitudes of that magnitude are not typically acceptable for aerospace shell structures used in primary structures.

\section{Response of a Cylinder with a Lateral Load Imperfection}

Following the approach of Hühne et al., ${ }^{13}$ a second type of imperfection was considered which used a lateral perturbation load $Q$ to create a local imperfection. As defined in Figure 2, the lateral perturbation load was applied at the mid-length of the cylinder, and for the purpose of discussion was assumed to be applied at $s=0.5 C$. Cylinder buckling loads, or critical loads, over a range of lateral perturbation loads $100 \leq Q \leq 2000 \mathrm{lbs}$ were determined. The critical load vs. lateral perturbation load response and the corresponding load vs. axial displacement responses are shown in Figs. 9 and 10 for the full-scale and subscale cylinders, respectively. The load vs. axial displacement responses corresponding to the bounding values of the lateral perturbation load are plotted with red lines, and the responses for all perturbation loads end at the critical load. However, as observed by Hühne et al., for a certain range of lateral perturbation loads, global buckling was preceded by a local buckling event. To illustrate the cylinder response between the local buckling event and global buckling, detailed views of the load vs. end displacement responses shown in Figs. 9b and 10b are magnified in Figs. 9c and 10c. In these subfigures, the critical loads are identified by the open black triangles, and for those cases where local buckling occurred, the local critical loads are identified by the filled blue triangles. The behavior of the cylinder during the local buckling event will be described subsequently.

There are three characteristic behaviors that were observed when determining the effect of the lateral perturbation load on the cylinder response, and the ranges over which these behaviors occurred were approximately the same for the full-scale and sub-scale cylinders. For lateral perturbation loads $100 \leq Q \leq 300 \mathrm{lbs}$ for the full-scale cylinder and $100 \leq Q \leq 400$ for the sub-scale cylinder, the cylinder was very sensitive to changes in the magnitude of the lateral perturbation load where the critical load decreased approximately $20 \%$ over this range as the perturbation load was increased. The corresponding buckling event was characterized by a general instability and significant loss of load carrying capacity of the cylinder. For perturbation loads within this range there was no change in the prebuckling axial stiffness of the cylinder, and the load vs. end displacement responses are coincident with the response of the perfect cylinder. The postbuckling responses for this range of perturbation loads exhibited different postbuckling displacement patterns depending on the magnitude of the perturbation load. There was little effect on the postbuckling configuration for small values of the perturbation load where the initial postbuckling configuration was similar to that of the perfect cylinder as shown in Figs. 3e and 4e. However, as the perturbation load was increased the local dimple began to influence the global buckling displacements by reducing the number of axial half waves from two axial half waves to one axial half wave centered at the point of application of the perturbation load. Over the ranges of lateral perturbation loads $400 \leq Q \leq 750 \mathrm{lbs}$ and $500 \leq Q \leq 700 \mathrm{lbs}$ for the full-scale and sub-scale cylinders, respectively, a local buckling event occurred and was characterized by a snap-through type buckling where the magnitude of the normal displacement of the dimple increased. In Figs. 9a and 10a the local buckling loads for the values of the lateral perturbation load where this dimple buckling occurred are identified with the filled blue triangles. An example of this local buckling event is shown for the full-scale cylinder with a lateral load of $Q=700 \mathrm{lbs}$ in Fig. 11. A magnified view of the load vs. end displacement response is shown in Fig. 11a, to show the of the small drop in load that occurred during the buckling event. This small drop in load corresponds to the increase in the normal displacement at the node at which the perturbation load is applied as shown in Fig. 11b. The shapes and out-of-plane, or normal, displacements before and after local buckling are shown in Figs. 11c and 11d, respectively. Within this range of lateral loads and after local buckling occurred, the cylinder was able to carry additional load until a critical load was obtained, corresponding to the global buckling load. The axial stiffness of the cylinder prior to local buckling was the same as the axial stiffness of the perfect cylinder. However, the effective axial stiffness of 
the shell in the local postbuckling response was approximately $12 \%$ less than the prebuckling axial stiffness. Overall, the cylinders that exhibited this initial local buckling behavior, exhibited postbuckling reductions in axial stiffness between $12 \%$ and $15 \%$ of the prebuckling stiffness for the full-scale cylinder and between $16 \%$ and $18 \%$ of the prebuckling stiffness for the sub-scale cylinder. While there was sensitivity to changes in the perturbation load when the local buckling occurred, there was little sensitivity for the global critical load within this range. The global critical loads for this range of lateral loads were all within $2 \%$ of each other. From $800 \leq Q \leq 2000 \mathrm{lbs}$, for both the full-scale and sub-scale cylinders, the critical load decreased approximately $4 \%$, and indicates only slight sensitivity to variations in the perturbation load. In addition, the postbuckling configuration of the cylinder is affected by the presence of the perturbation load. For example the response of the full-scale cylinder with a lateral perturbation load of $Q=800 \mathrm{lbs}$ is shown in Fig. 12. In Figs. 12a and $12 \mathrm{~b}$ the black triangle and blue diamond denote the critical load and initial postbuckling configurations, respectively, which are shown in Figs. 12c and 12d. When the cylinder buckled, there was only a small change in the dimple/buckle created by the lateral load, while similar, but smaller, single axial half wave buckles developed around the circumference of the cylinder and is in contrast to the global buckling behavior exhibited in Figs. 3 and 4 for the perfect cylinder. Changes in the normal displacement at the point of application of the lateral load, $s=0.5 C$, and its diametric counterpart, at $s=0$, shown in Fig. 12b, reiterate this point. Sub-scale cylinders exhibited similar postbuckling behavior for this range of lateral loads. The behavior described above was similar to that observed by Hühne et al. for unstiffened thin-walled composite cylinders. Based on the predicted critical loads and considering the effect of the lateral perturbation load on the critical load, Figs. 9a and 10a, lower bounds for the buckling load were established for lateral perturbation loads at the threshold value where the cylinder changes from being very sensitive to changes in the perturbation load to being insensitive. The perturbation loads at which these threshold values occurred and the corresponding critical loads were $Q=400 \mathrm{lbs}$ and $P^{c r}=0.77 P_{\text {full }}^{c r, p e r}$ for the full-scale cylinder and $P^{c r}=0.79 P_{\text {sub }}^{c r, p e r}$ for the sub-scale cylinder.

\section{Response of a Cylinder with a Single Dimple Imperfection}

One question regarding using the lateral load perturbation as an imperfection is what effect the perturbation load has on the response, more specifically, how the local stresses affect the response since it is likely that any real geometric imperfection might be relatively stress-free. To investigate this, a single dimple imperfection, that is based on the deformed shape of a cylinder subjected to a lateral perturbation load and no axial end displacement, was considered. Specifically, the deformed shape of a cylinder with $Q=1000 \mathrm{lbs}$ and $\delta=0$ determined using a nonlinear static analysis was used as the single dimple imperfection. This perturbation load was chosen because it was greater than the threshold value and in a range where the critical load of the cylinder was insensitive to changes in the perturbation load. The same lateral perturbation load was used for the full-scale and sub-scale cylinders.

A subroutine in STAGS was used to convert the resulting displacements for this load case into an imperfection. After extracting the translational displacements from the load case described above, the displacements were normalized by the largest magnitude of the normal displacement $w_{\max }$, the normalized displacements were then multiplied by an imperfection amplitude $a$, and this shape was applied to the perfect geometry as an initial geometric imperfection. The imperfection shapes are shown in Fig. 13. For the full-scale cylinder, the dimple spanned an arc length of $s \approx 0.043 C$ which was approximately 5.3 orthogrid pockets. For the sub-scale cylinder, the dimple spanned an arc length of $s \approx 0.056 C$ which was approximately 5.6 orthogrid pockets. Imperfection amplitudes over the range $0.010 \leq a / H \leq 1.00$ were considered in this study.

For the full-scale cylinder, the effect of the imperfection amplitude on the critical load for the single dimple imperfection is shown in Fig. 14 along with the corresponding load vs. end displacement responses. The analogous plots for the sub-scale cylinder are shown in Fig. 15. Detailed views of the load vs. end displacement responses are shown in Figs. 14c and 15c, and symbols are used to identify the critical load for each imperfection amplitude considered.

The behavior of the cylinders with the single dimple imperfection was very similar to the behavior of cylinders with the lateral load imperfection. There were three ranges of imperfection amplitudes over which different behaviors were observed, and the types of behavior were similar to that for the lateral perturbation load imperfection. In the first range of imperfection amplitudes of $0.010<a / H<0.220$ for the full-scale cylinder and $0.010<a / H<0.18$ for the sub-scale cylinder the critical load was sensitive to changes in the imperfection amplitude. There was no change in the axial stiffness of the cylinders with imperfection 
amplitudes within this range. In the second range of imperfection amplitudes, $0.24<a / H<0.40$ for the full-scale cylinder and $0.190<a / H<0.28$ for the sub-scale cylinder, there was a local snap-through buckling of the single dimple, then additional axial end displacement was applied to the shell and the global buckling load was obtained. The local limit loads are plotted with the filled blue diamonds in Figs. 14a and 15a and Figs. 14c and 15c. There was no change in the axial stiffness of the cylinders prior to local buckling, but after buckling, the stiffness was was reduced by approximately $20 \%$ and $23 \%$ for the full-scale and sub-scale cylinders, respectively. In the third range of imperfection amplitudes, $0.60<a / H<1.0$ for the full-scale cylinder and $0.30<a / H<1.0$ for the sub-scale cylinder, the behavior was similar to what was observed in the last range of lateral perturbation loads in that the there was no local buckling and the initial postbuckling configuration, characterized by one axial half-wave buckles, was similar to the configuration shown in Fig. 12d. Over the ranges $0.24<a / H<1.0$ for the full-scale cylinder and $0.19<a / H<1.0$ for the sub-scale cylinder, the critical loads were nearly identical. The constant loads over these ranges established lower bounds at $P^{c r}=0.78 P_{\text {full }}^{c r, p e r}$ and $P^{c r}=0.80 P_{\text {sub }}^{c r, p e r}$ for the full-scale and sub-scale cylinders, respectively.

\section{E. Comparison of Lower Bound Buckling Loads}

In the preceding sections, the effects of three imperfection types on cylinder response were discussed. For the geometric imperfections, mode shape and single dimple, the effect of imperfection amplitude on the critical axial load are compared in Fig. 16. For the lateral perturbation load imperfection, the imperfection amplitude was assumed to be the normal displacement at the node where the lateral load was applied with no applied axial end displacement, and these results are also shown in Fig. 16. For each imperfection type, the lower bound predictions and the imperfection amplitude at which the lower bound was established are compiled in Table 2. For the lateral perturbation load imperfection and the single dimple imperfection, the imperfection amplitude used to establish the lower bound was taken as the value located at the transition between amplitude-sensitive and amplitude-insensitive behaviors. For the mode shape imperfection, the lower bound was established at a local minimum in the critical axial load vs. imperfection amplitude data.

Lower bounds predicted using the lateral perturbation load and single dimple imperfections are within $2 \%$ of each other for both the full-scale and sub-scale cylinders, and indicate that local stresses associated with the dimple have a small effect on the overall behavior. Lower bounds predicted using the mode shape imperfection are $20 \%$ and $18 \%$ lower than lower bound predicted using the lateral perturbation load imperfection for the full-scale and sub-scale cylinders, respectively. The mode shape imperfection also had a greater effect on the axial stiffness of

Table 2. Predicted lower bounds and imperfection amplitude at which lower bound was established

\begin{tabular}{|c|c|c|c|c|}
\hline & \multicolumn{2}{|c|}{ full-scale } & \multicolumn{2}{c|}{ sub-scale } \\
\hline $\begin{array}{c}\text { imperfection } \\
\text { type }\end{array}$ & $\begin{array}{c}\text { lower } \\
\text { bound, } \\
\frac{P^{c r}}{P_{\text {furper }}^{c r, p e r}}\end{array}$ & $\begin{array}{c}\text { imperfection } \\
\text { amplitude, } \\
\frac{a}{H}\end{array}$ & $\begin{array}{c}\text { lower } \\
\text { bound, } \\
\frac{P^{c r}}{P_{\text {sub }}^{c r p r}}\end{array}$ & $\begin{array}{c}\text { imperfection } \\
\text { amplitude, } \\
\frac{a}{H}\end{array}$ \\
\hline mode shape & 0.63 & 0.20 & 0.66 & 0.140 \\
\hline $\begin{array}{c}\text { lateral } \\
\text { perturbation } \\
\text { load }\end{array}$ & 0.77 & 0.22 & 0.79 & 0.157 \\
\hline $\begin{array}{c}\text { single } \\
\text { dimple }\end{array}$ & 0.78 & 0.24 & 0.80 & 0.190 \\
\hline
\end{tabular}
the cylinder than either of the other two imperfection types, and is a reduction in stiffness that is not typically seen in actual structural testing. Determining a lower bound for the mode shape imperfection was not as straightforward as for either the lateral perturbation load or single dimple imperfections. There was much more variability in the effect of the imperfection amplitude for the mode shape imperfection. Although not considered in this study, other mode shape imperfections could have a significantly different effect when predicting a lower bound, one that could be more or less conservative than the one mode shape that was considered herein. Lower bounds predicted for the full-scale and sub-scale cylinders were within $3 \%$ for the lateral perturbation load and single dimple imperfections, and within 5\% for the mode shape imperfection. Small differences would be expected between the two cylinders sizes because the sub-scale cylinder was not scaled down exactly from the full-scale cylinder. 


\section{Concluding Remarks}

Numerically predicted responses of imperfect orthogrid-stiffened circular cylinders subjected to an axial compressive load have been compared to the response of a geometrically perfect cylinder. Two cylinder sizes were considered, a full-scale cylinder of a size typical of a space launch vehicle, and a sub-scale cylinder of a size more practical for testing in a laboratory. The three types of imperfections used to predict lowerbound buckling loads for axially compressed orthogrid-stiffened circular cylinders included a mode shape imperfection calculated from a linear bifurcation buckling analysis, a lateral perturbation load imperfection, and a single dimple imperfection with the initial geometric shape based on the shape resulting from a lateral perturbation load imperfection. Each imperfection type was used to predict a lower bound buckling load, and a comparison was made between the three lower bounds.

The lateral perturbation load and a single dimple imperfection predict a similar lower bound buckling load, and one that is significantly larger than that predicted using the mode shape imperfection that was considered. In terms of establishing a lower bound, the effect of the local stresses that result from the application of the perturbation load were minimal when compared to the lower bound established using a stress-free single dimple imperfection. The effect of cylinder size on the lower bound predictions was minimal for all three imperfection types. If proper validation can be accomplished for the lower bound predicted using the lateral perturbation load method, this approach could help to reduce unnecessary conservatism in launch vehicle design that could result from using a more traditional approach like using the mode shape imperfection to predict a lower bound design value.

\section{References} 1965.

${ }^{1}$ Anon., "Buckling of Thin-Walled Circular Cylinders." NASA Space Vehicle Design Criteria, NASA SP-8007, September

${ }^{2}$ Koiter, W. T., On the Stability of Elastic Equilibrium, (in Dutch), H. J. Paris, Amsterdam, Holland, 1945, translation available as AFFDL-TR-70-25, February 1970, Wright-Patterson Air Force Base.

${ }^{3}$ von Kármán, T. and Tsien, H.-S., "The Buckling of Thin Cylindrical Shells Under Axial Compression," Journal of the Aeronautical Sciences, Vol. 8, No. 8, 2008, pp. 301-312.

${ }^{4}$ Budiansky, B. and Hutchinson, J., "Dynamic Buckling of Imperfection Sensitive Structures," Proceedings of the 11th IUTAM Congress, edited by H. Gortler, Springer-Verlag, Berlin, 1964, pp. 636-651.

${ }^{5}$ Árbocz, J. and Babcock, C. D., "The Effect of General Imperfections on the Buckling of Cylindrical Shells," Journal of Applied Mechanics, Vol. 36, No. 1, 1969, pp. 28-38.

${ }^{6}$ Sechler, E. E., "The Historical Development of Shell Research and Design," Thin-Shell Structures, Theory, Experiments, and Design, edited by Y. C. Fung and E. E. Sechler, Prentice-Hall, Englewood Cliffs, NJ, 1974, pp. 3-25.

${ }^{7}$ Árbocz, J., "The Effects of Imperfect Boundary Conditions on the Collapse Behavior of Anisotropic Shells," Proceedings of the Joint Applied Mechanics and Materials ASME Summer Conference, AMD-MD‘95, Los Angeles, CA, June 28-30 1995.

${ }^{8}$ Árbocz, J., Starnes, J. H., Jr., and Nemeth, M. P., "A Hierarchical Approach to Buckling Load Calculations," Proceedings of the 40th AIAA/ASME/ASCE/AHS/ASC Structures, Structural Dynamics, and Materials Conference, St. Louis, MO, April 1999, AIAA Paper 1999-1232.

${ }^{9}$ Koiter, W. T., Elishakoff, I., Li, Y. W., and Starnes, J. H., Jr., "Buckling of an Axially Compressed Imperfect Cylindrical Shell of Variable Thickness," Proceedings of the 35th AIAA/ASME/ASCE/AHS/ASC Structures, Structural Dynamics, and Materials Conference, Hilton Head, SC, April 1994, AIAA Paper 1994-1339.

${ }^{10}$ Starnes, J. H., Jr., Hilburger, M. W., and Nemeth, M. P., "The Effects of Initial Imperfections on the Buckling of Composite Shells," Composite Structures: Theory and Practice, ASTM STP 1383, edited by P. Grant and C. Q. Rousseau, American Society for Testing and Materials, 2000, pp. 529-550.

${ }^{11}$ Hilburger, M. W. and Starnes, J. H., Jr., "Effects of Imperfections on the Buckling Response of Compression-Loaded Composite Shells," Proceedings of the 41st AIAA/ASME/ASCE/AHS/ASC Structures, Structural Dynamics, and Materials Conference, Atlanta, GA, April 2000, AIAA Paper 2000-1387.

${ }^{12}$ Holst, J. M., Rotter, J. M., and Calladine, C. R., "Imperfections in Cylindrical Shells Resulting from Fabrication Misfits," Journal of Engineering Mechanics, Vol. 125, No. 4, 1999, pp. 410-418.

${ }^{13}$ Hühne, C., Rolfes, R., Breitbach, E., and Teßmer, J., "Robust Design of Composite Cylindrical Shells under Axial Compression - Simulation and Validation," Thin-Walled Structures, Vol. 46, 2008, pp. 947-962.

${ }^{14}$ Rankin, C. C., Brogan, F. A., Loden, W. A., and Cabiness, H. D., "STAGS Users Manual, Version 5.0," Report LMSC P032594, Lockheed-Martin Missiles \& Space Co., March 1999.

${ }^{15}$ Riks, E., "Progress in Collapse Analysis," Journal of Pressure Vessel Technology, Transactions of the ASME, Vol. 109, No. 1,1987 , pp. 27-41. 


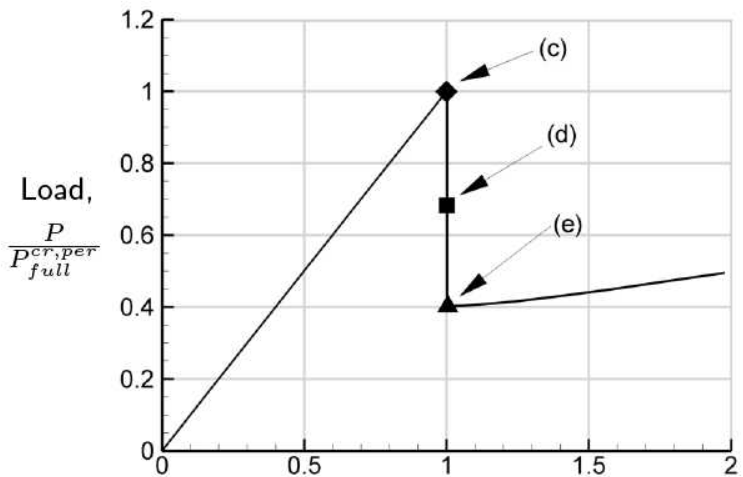

Axial End Displacement, $\frac{\delta}{\delta_{\text {full }}^{\text {cr,per }}}$

(a) load - end displacement relationship

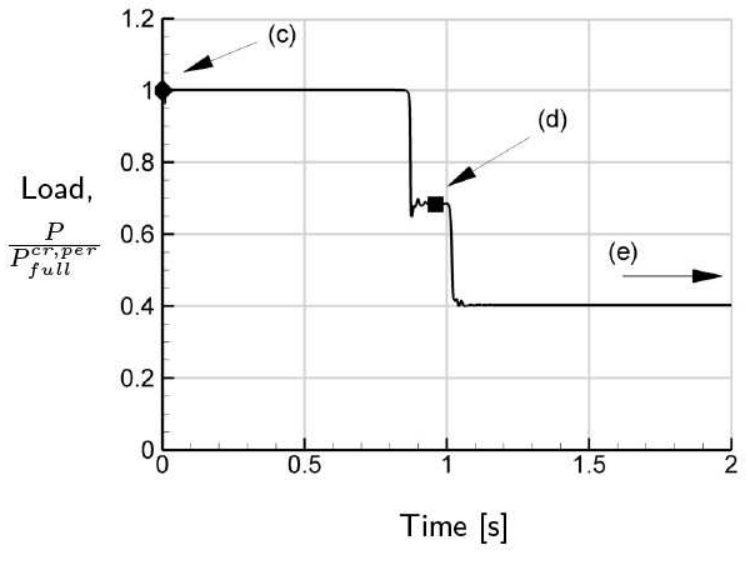

(b) load history during buckling
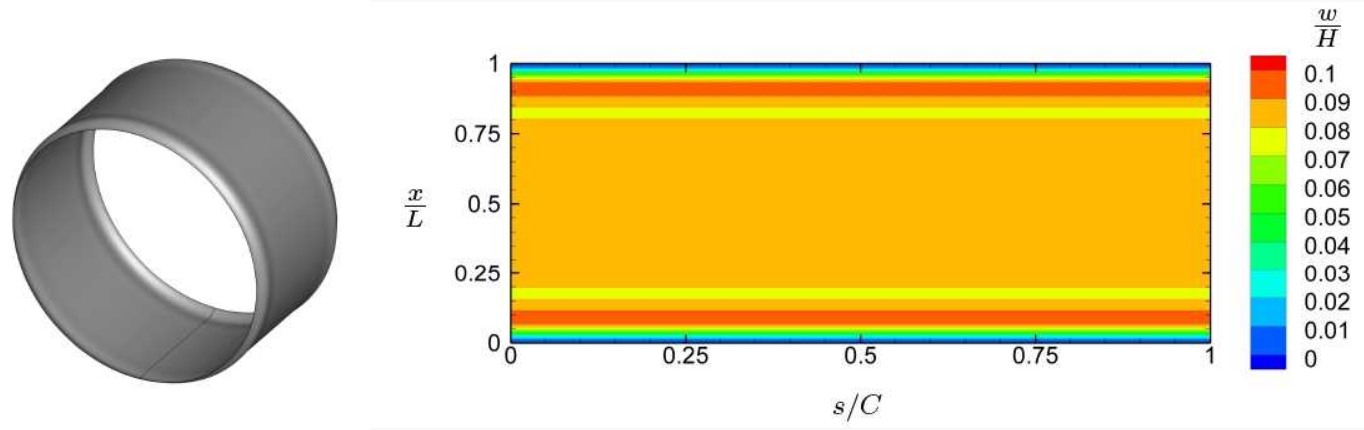

(c) shape and normal deformation for prebuckling configuration
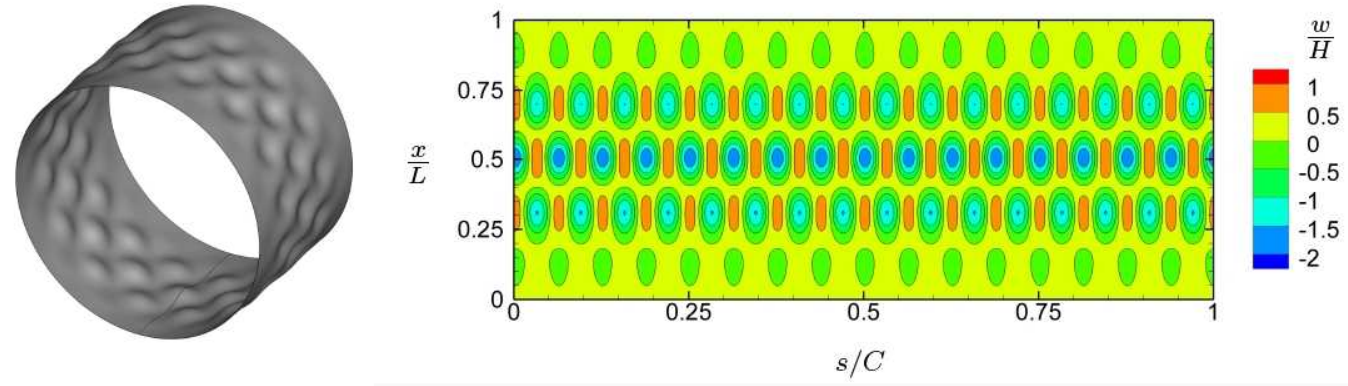

(d) shape and normal deformation for intermediate buckling configuration
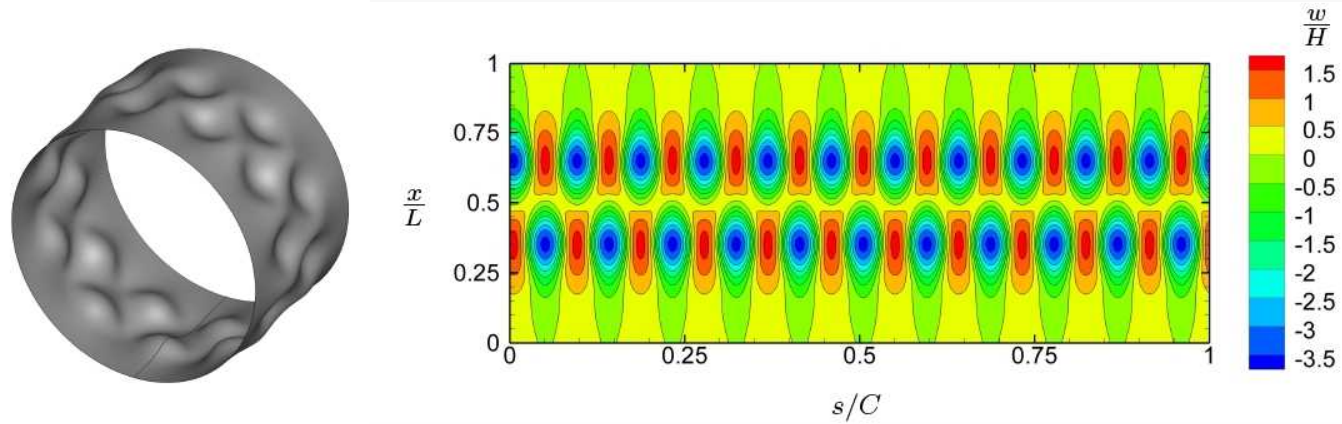

(e) shape and normal deformation for initial postbuckling configuration

Figure 3. Response of geometrically perfect full-scale cylinder 


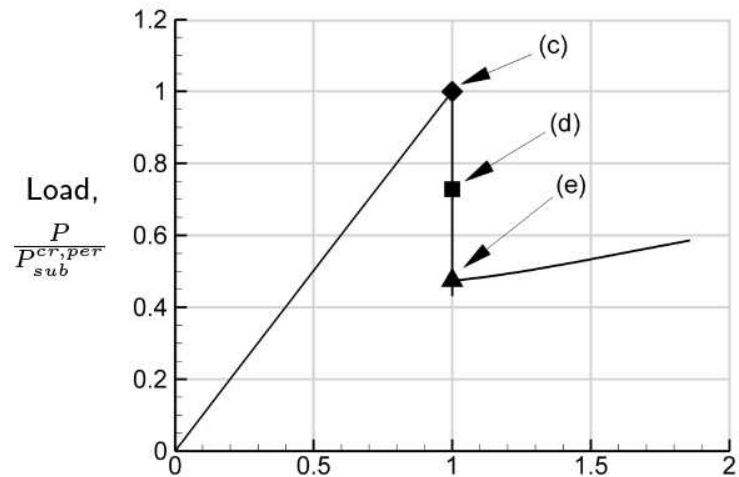

Axial End Displacement, $\frac{\delta}{\delta_{s u b}^{c r, p e r}}$

(a) load - end displacement relationship

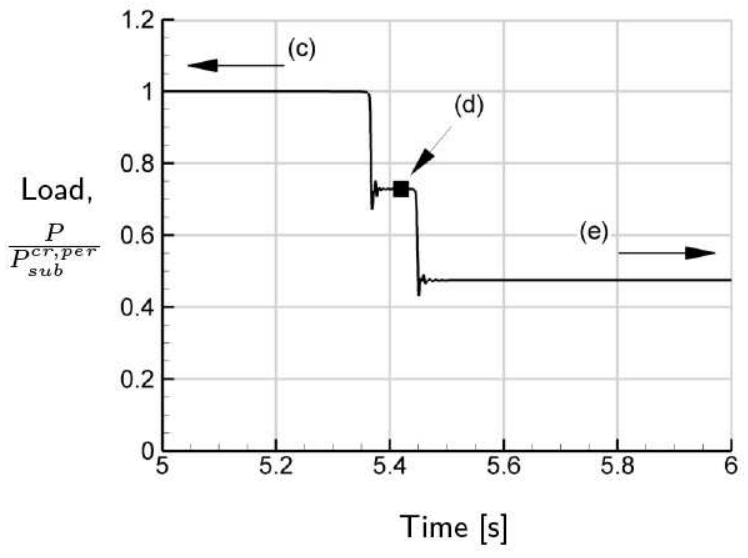

(b) load history during buckling
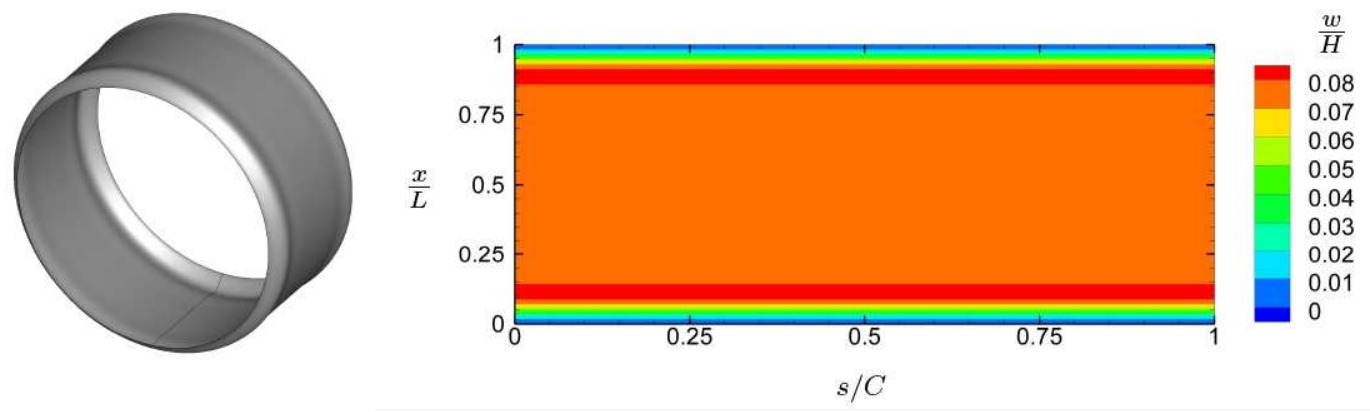

(c) shape and normal deformation for prebuckling configuration
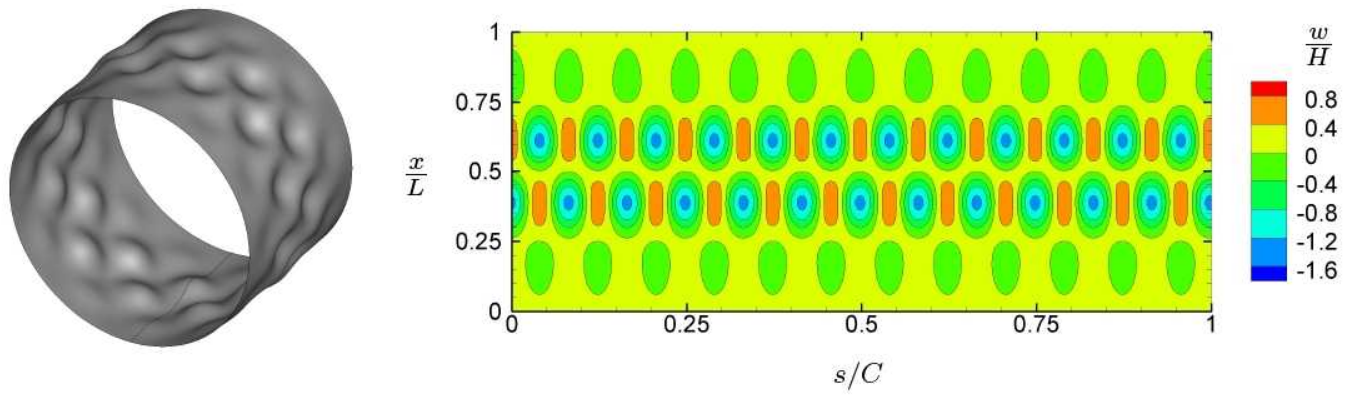

(d) shape and normal deformation for intermediate buckling configuration
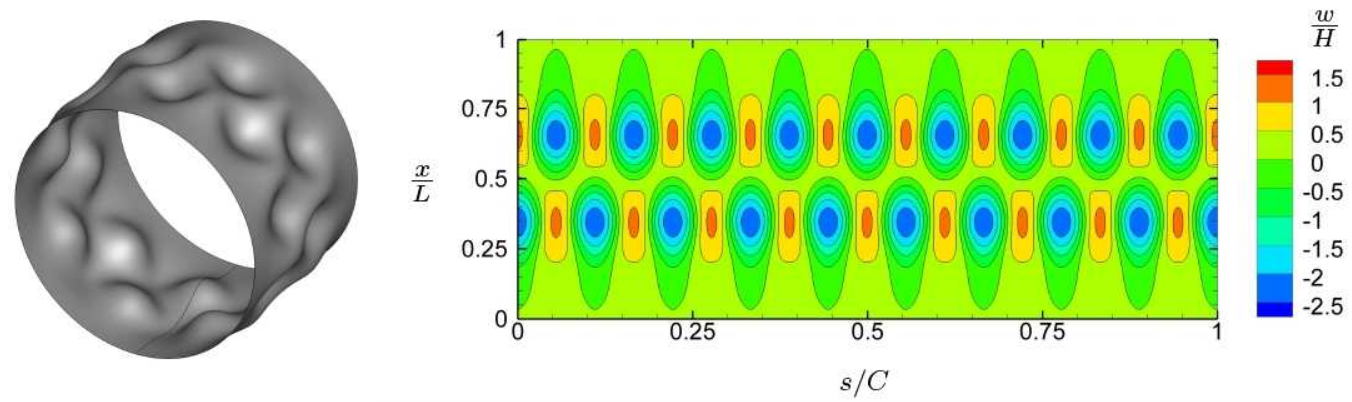

(e) shape and normal deformation for initial postbuckling configuration

Figure 4. Response of geometrically perfect sub-scale cylinder 

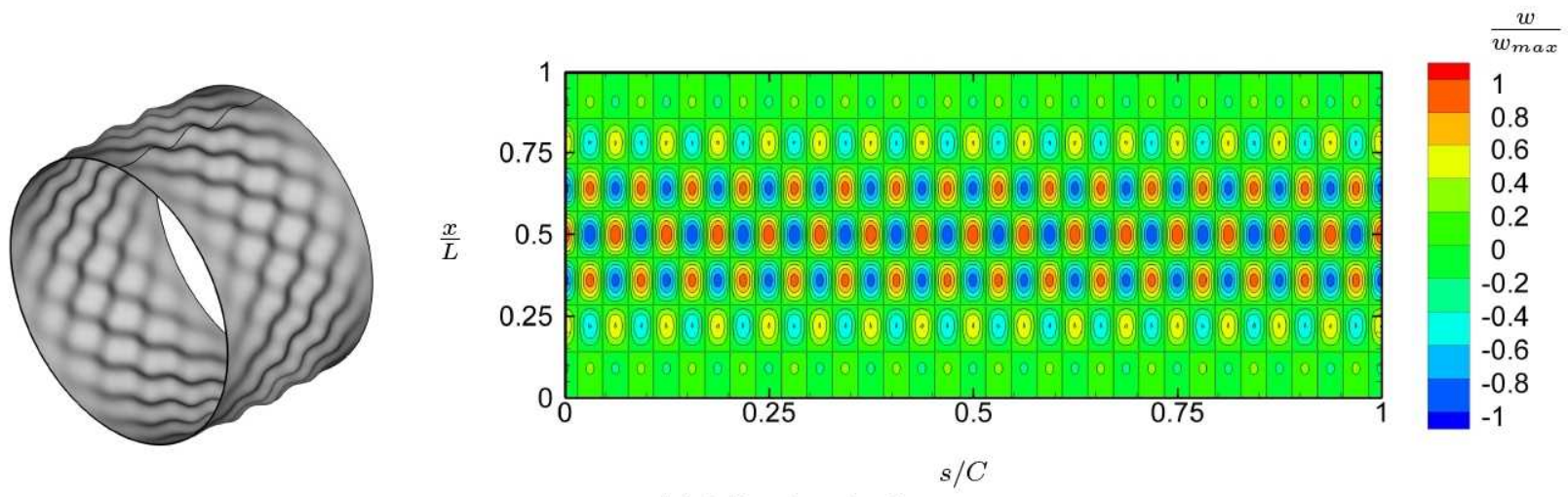

(a) full-scale cylinder
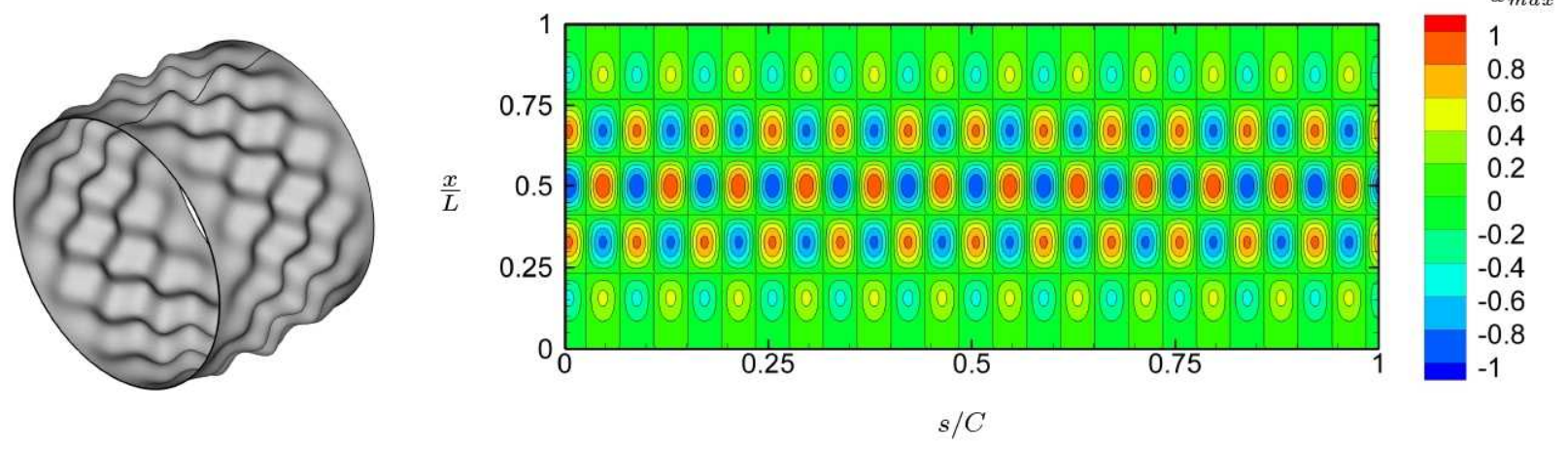

(b) sub-scale cylinder

Figure 5. Mode shape of first eigenvalue from bifurcation analysis

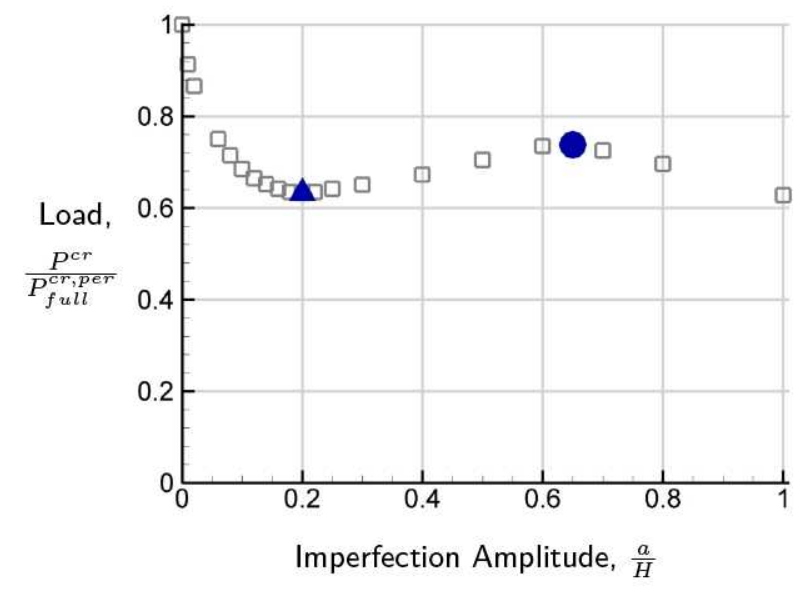

(a) maximum load - imperfection amplitude relationship

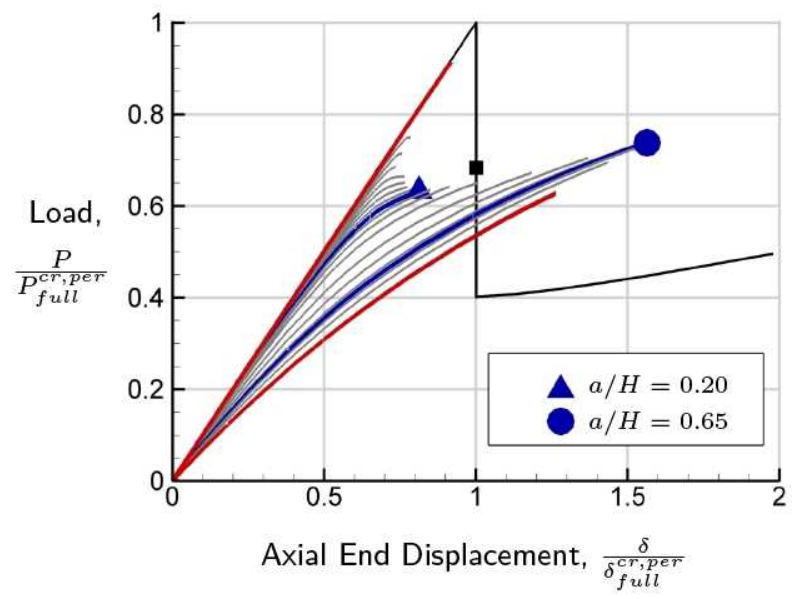

(b) load - end displacement responses for different imperfection amplitudes

Figure 6. Effect of mode shape imperfection amplitude on full-scale cylinder response 


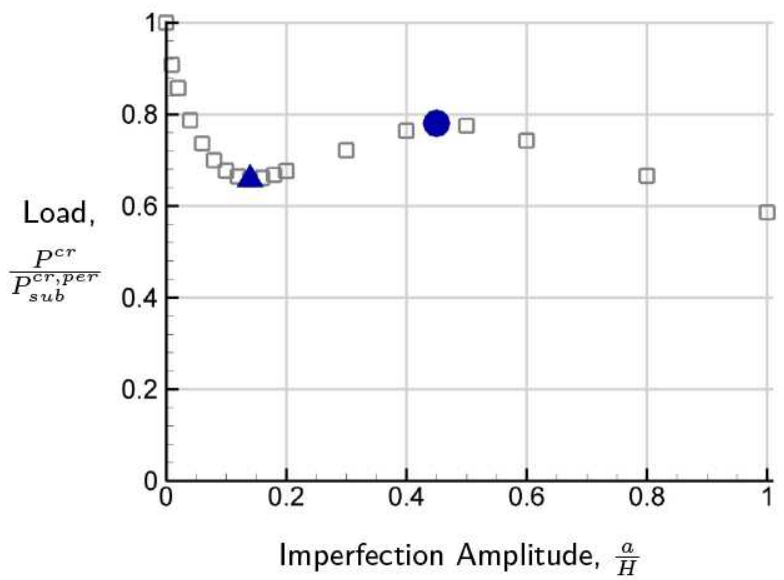

(a) maximum load - imperfection amplitude relationship

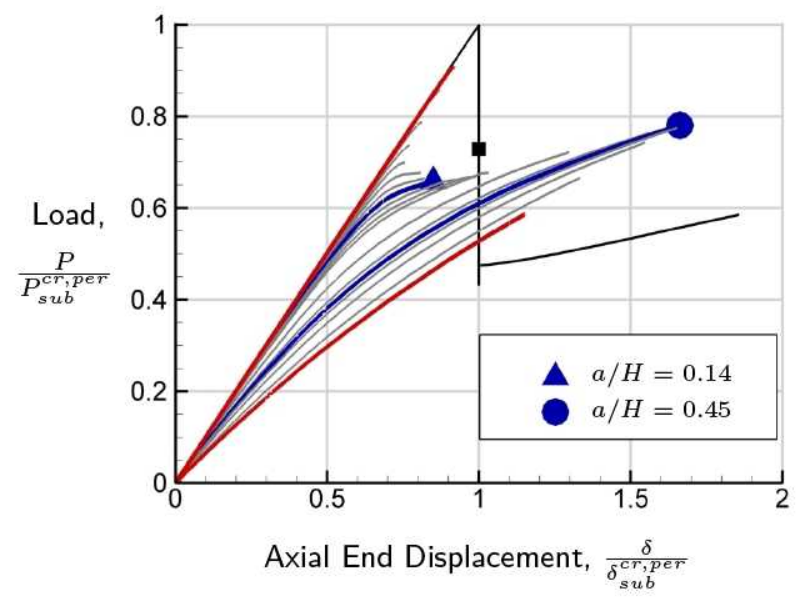

(b) load - end displacement responses for different imperfection amplitudes

Figure 7. Effect of mode shape imperfection amplitude on sub-scale cylinder response

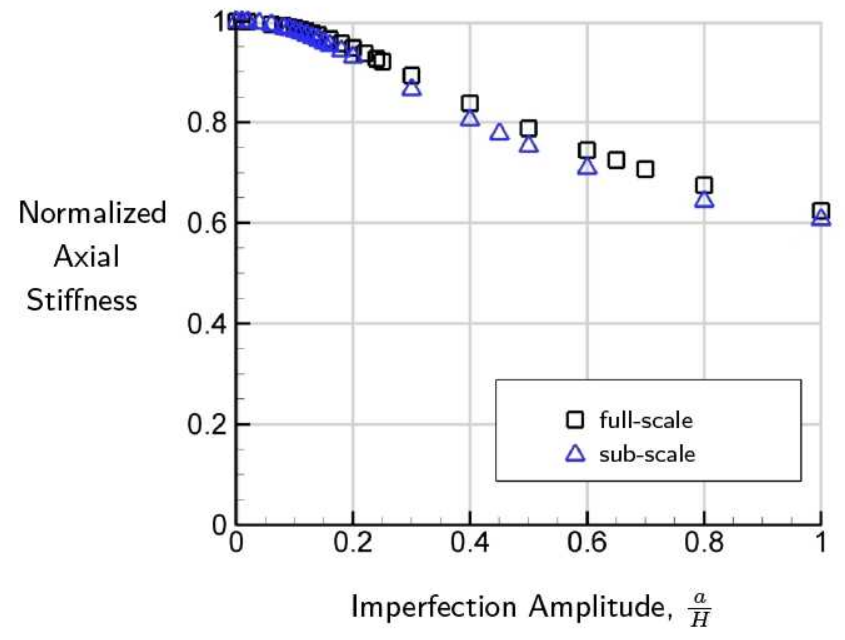

Figure 8. Effect of mode shape imperfection amplitude on effective cylinder axial stiffness 


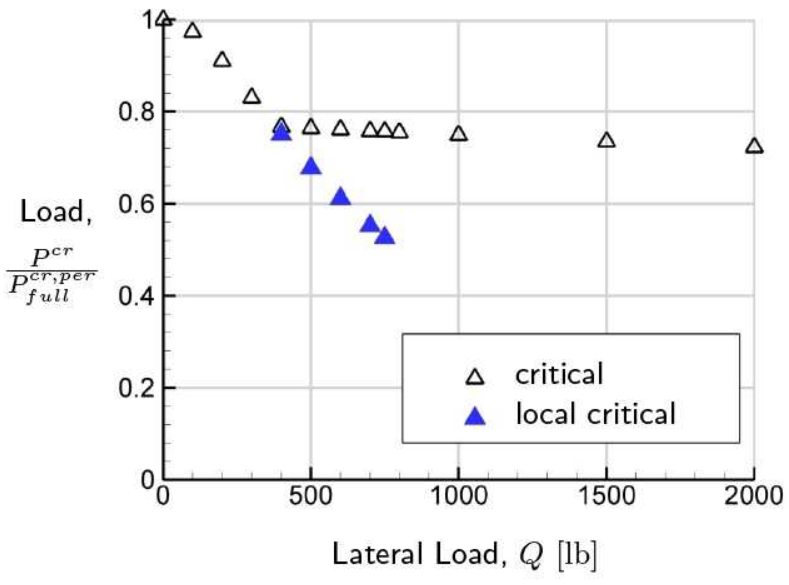

(a) critical load - imperfection amplitude relationship

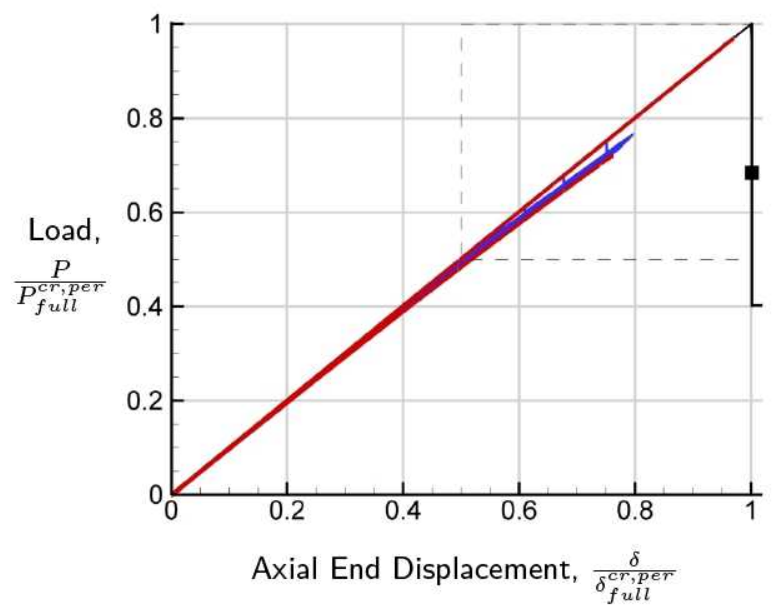

(b) load - end displacement responses for different imperfection amplitudes

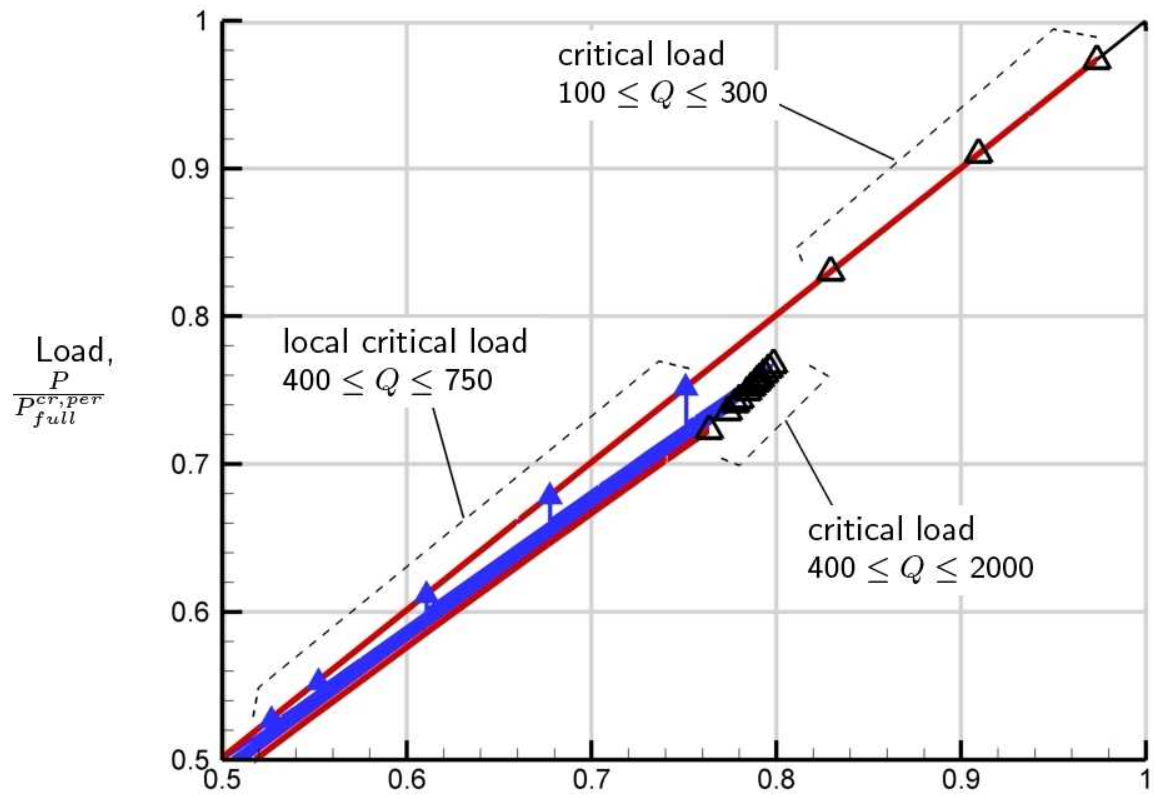

Axial End Displacement, $\frac{\delta}{\delta_{f u l l}^{c r, p e r}}$

(c) load - end displacement responses for different imperfection amplitudes

Figure 9. Effect of lateral perturbation load imperfection on full-scale cylinder response 


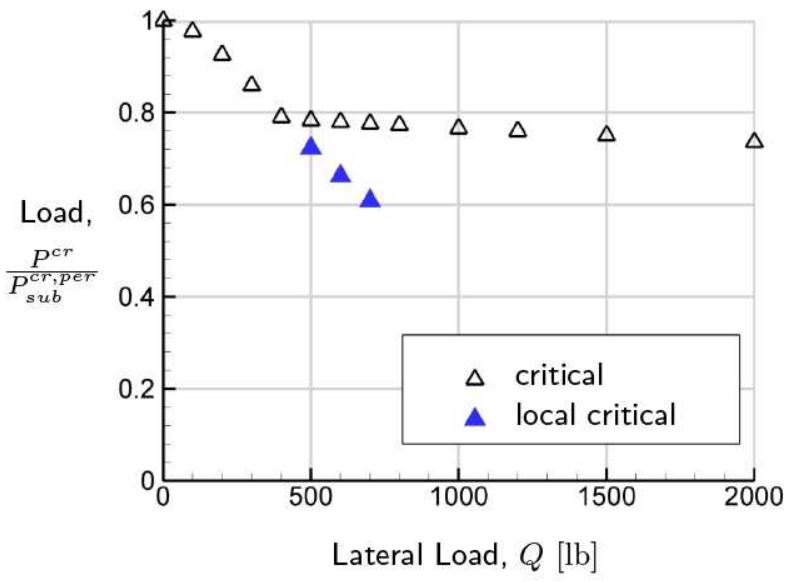

(a) critical load - imperfection amplitude relationship

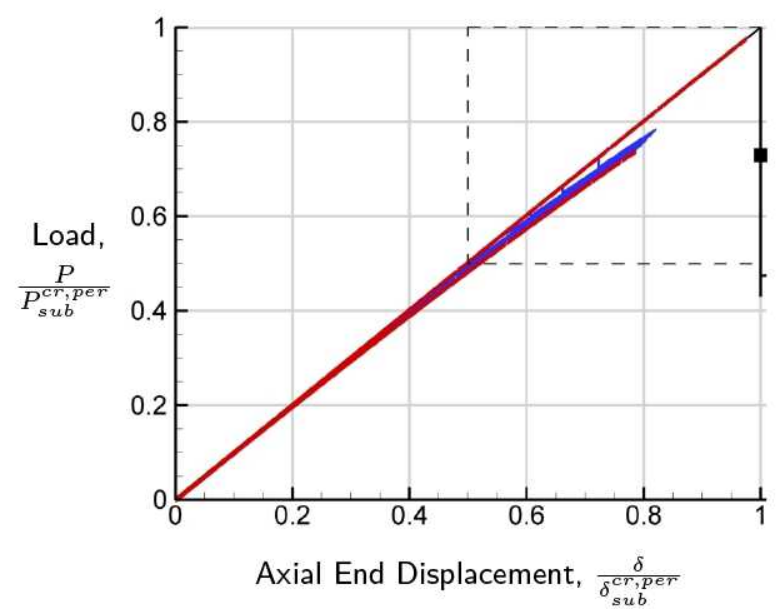

(b) load - end displacement responses for different imperfection amplitudes

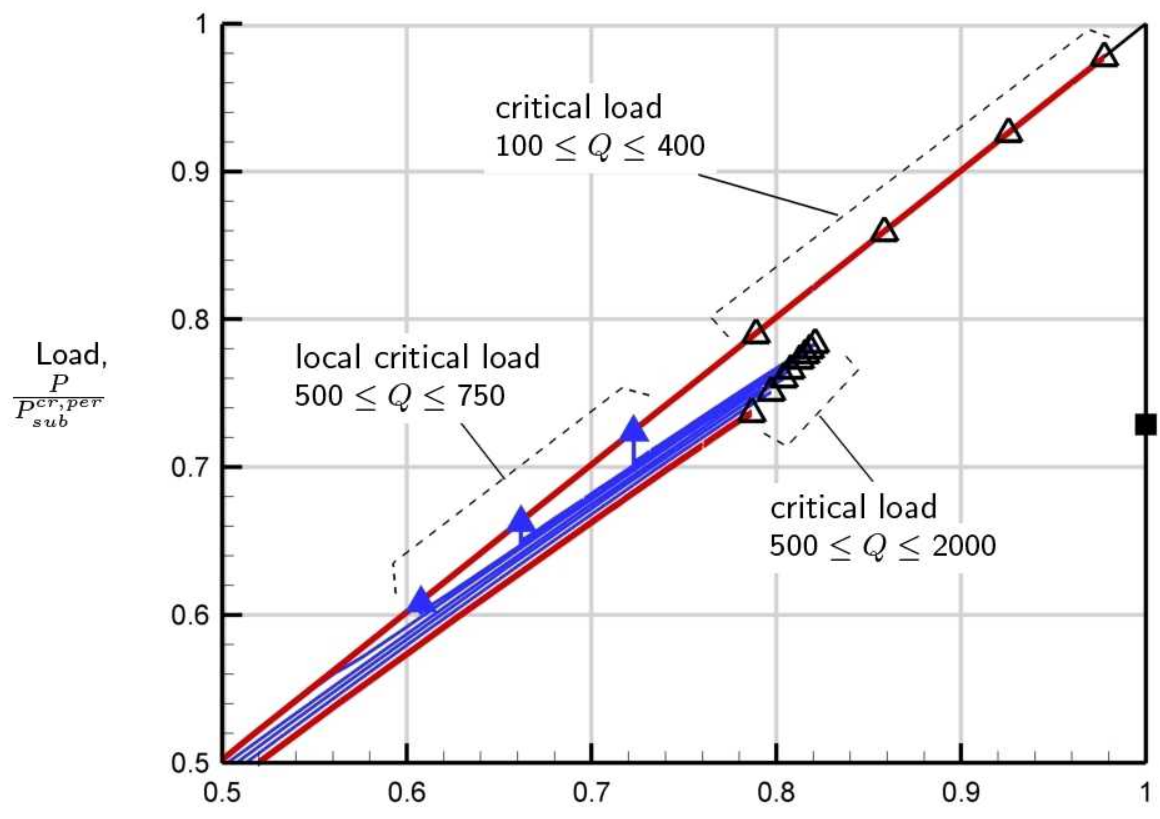

Axial End Displacement, $\frac{\delta}{\delta_{\text {sub }}^{\text {sr,per }}}$

(c) load - end displacement responses for different imperfection amplitudes

Figure 10. Effect of lateral perturbation load imperfection on sub-scale cylinder response 


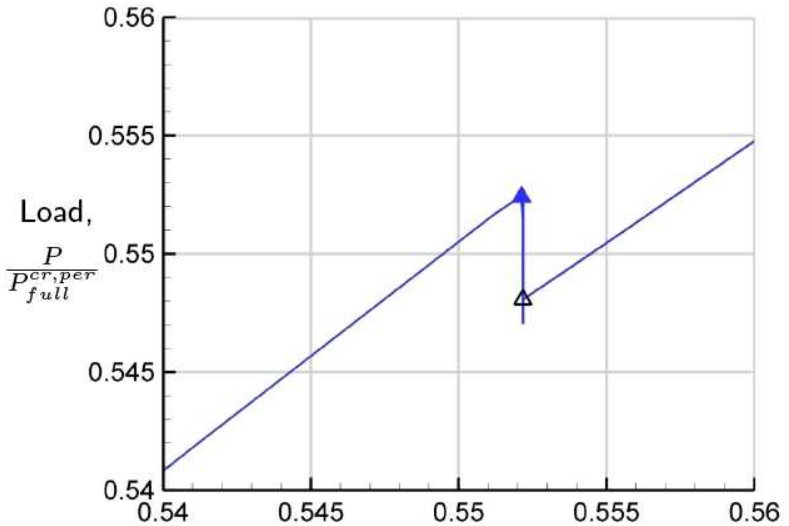

Axial End Displacement, $\frac{\delta}{\delta_{\text {full }}^{c r, p e r}}$

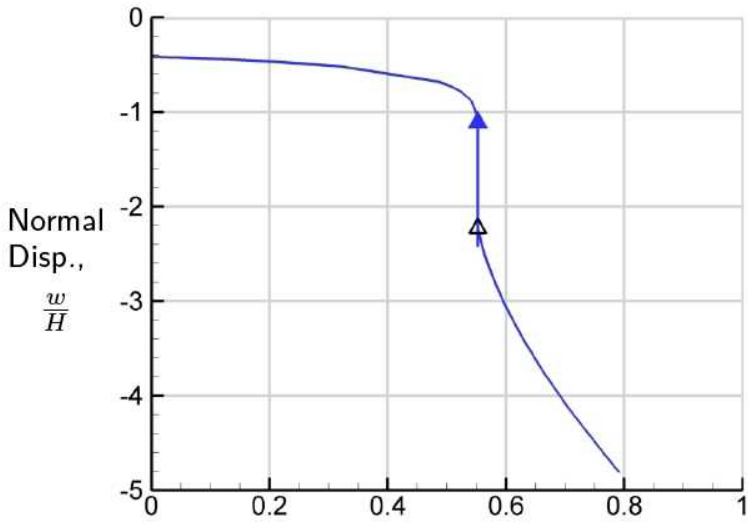

Axial End Displacement, $\frac{\delta}{\delta_{f u l l}^{c r, p e r}}$

(b) normal displacement vs. load response of point where lateral load perturbation is applied
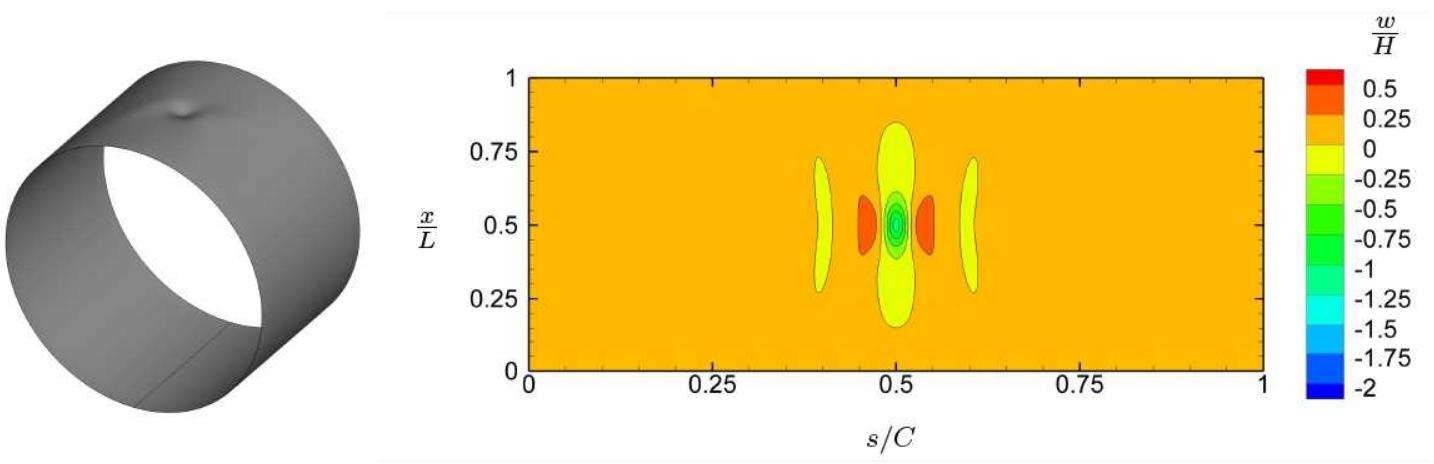

(c) shape and normal deformation for prebuckling configuration
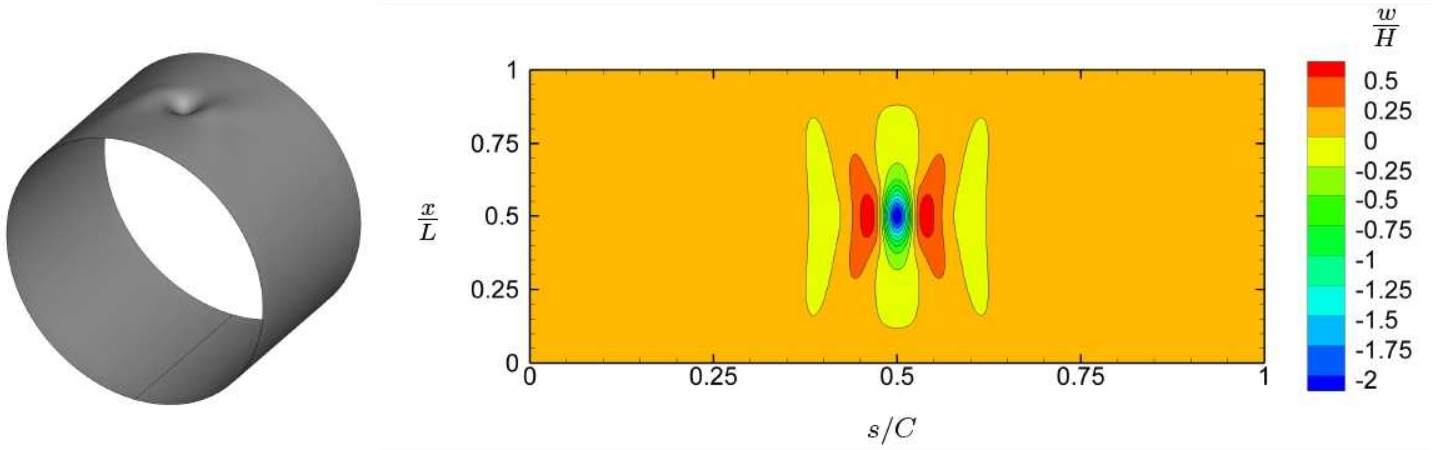

(d) shape and normal deformation for postbuckling configuration

Figure 11. Local buckling of full-scale cylinder with lateral perturbation load $\mathrm{Q}=\mathbf{7 0 0}$ lbs 


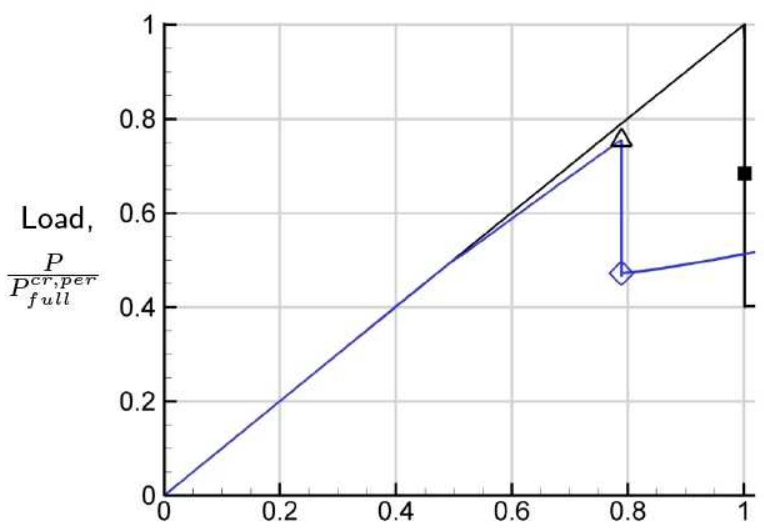

Axial End Displacement, $\frac{\delta}{\delta_{\text {full }}^{\text {cr,per }}}$

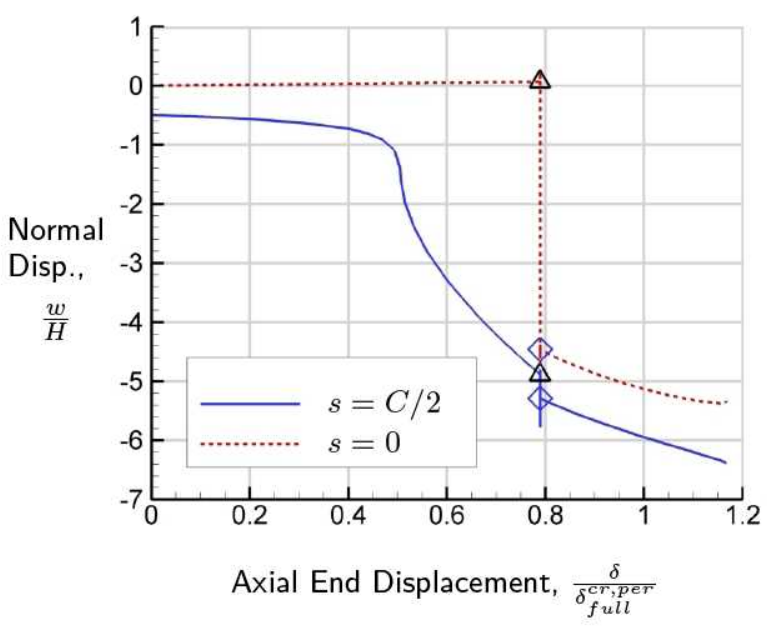

(b) normal displacement vs. load response

(a) load vs. end displacement response
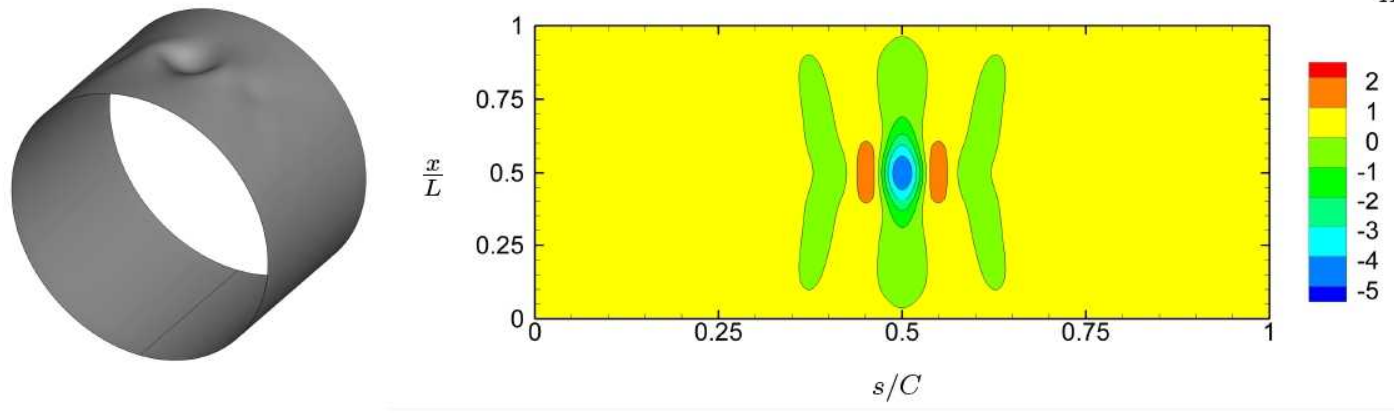

(c) shape and normal deformation for prebuckling configuration
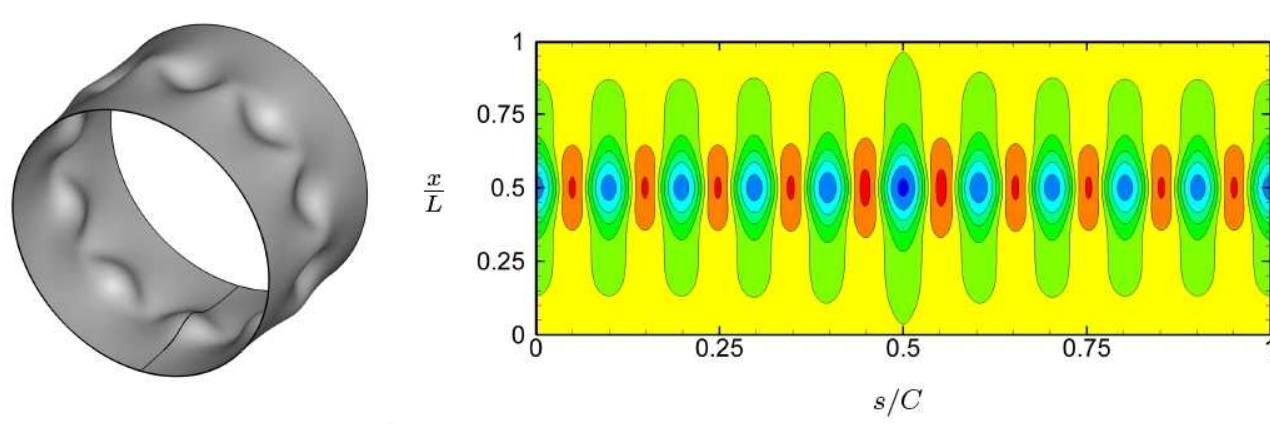

$\frac{w}{H}$

(d) shape and normal deformation for postbuckling configuration

Figure 12. Buckling of full-scale cylinder with lateral perturbation load $\mathrm{Q}=800 \mathrm{lbs}$ 

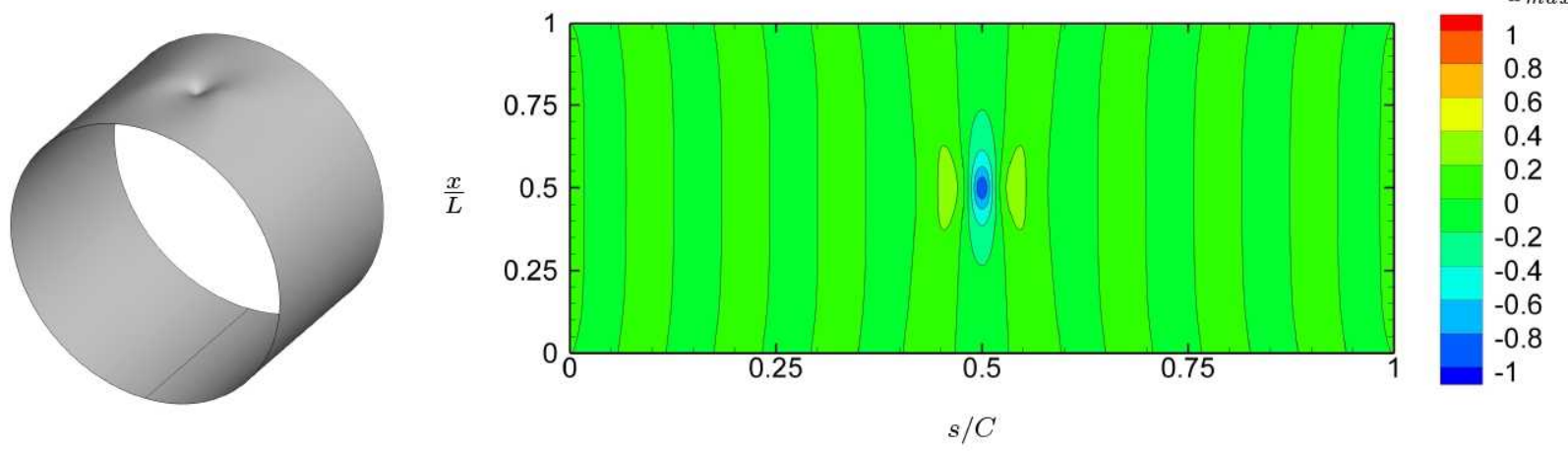

(a) full-scale cylinder
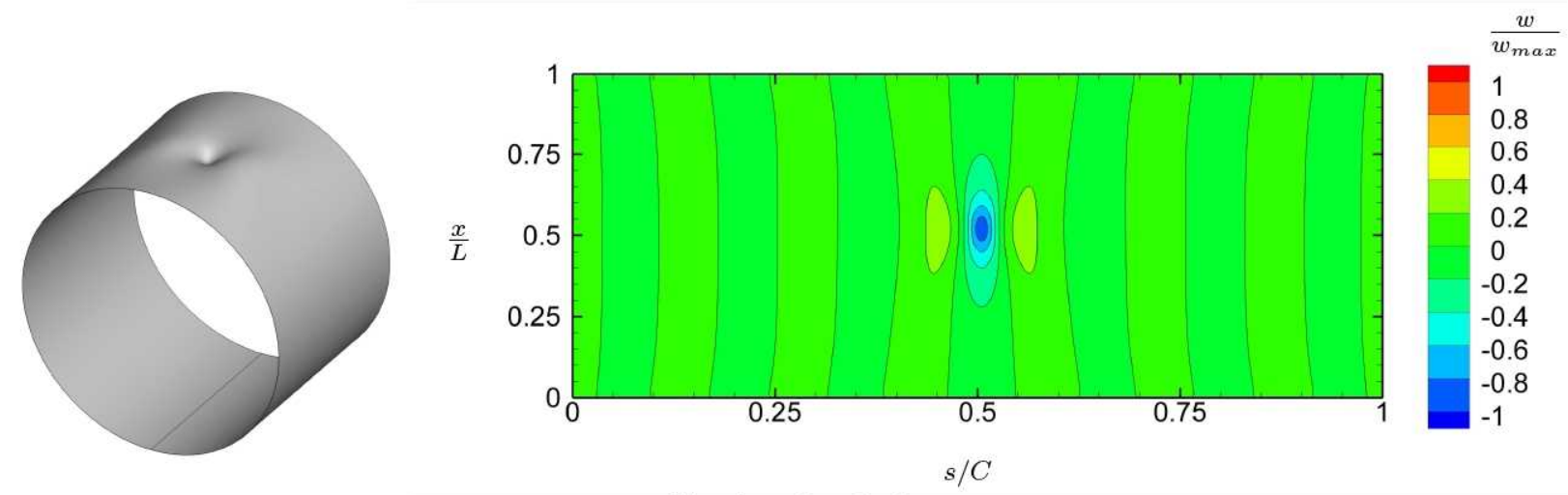

(b) sub-scale cylinder

Figure 13. Shape and normalized radial displacement used as basis for single dimple imperfection 


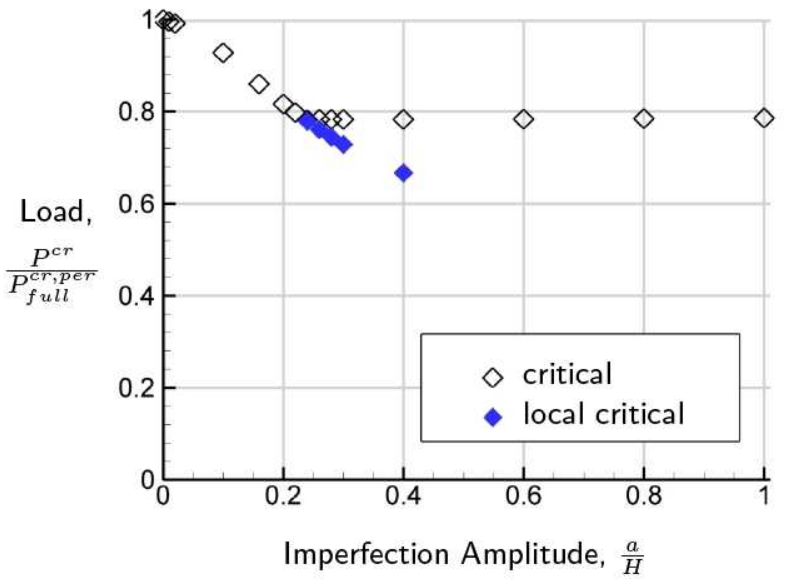

(a) critical load - imperfection amplitude relationship

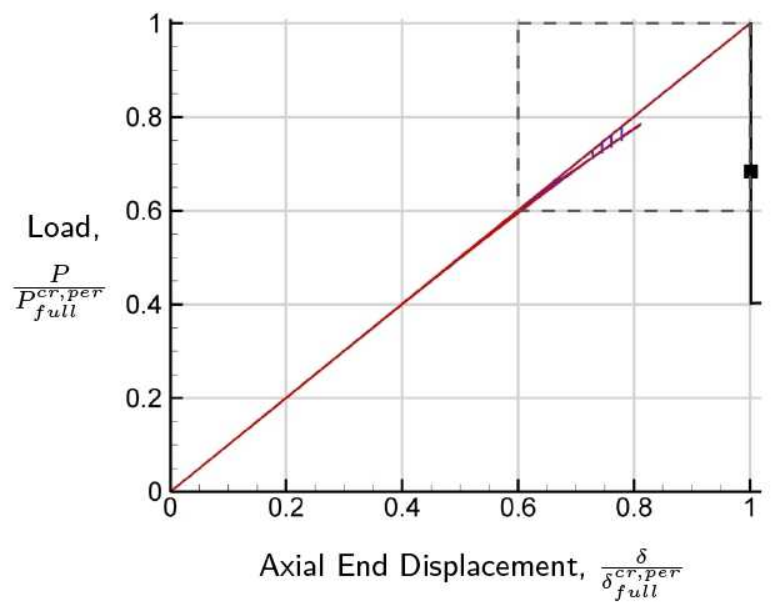

(b) load - end displacement responses for different imperfection amplitudes

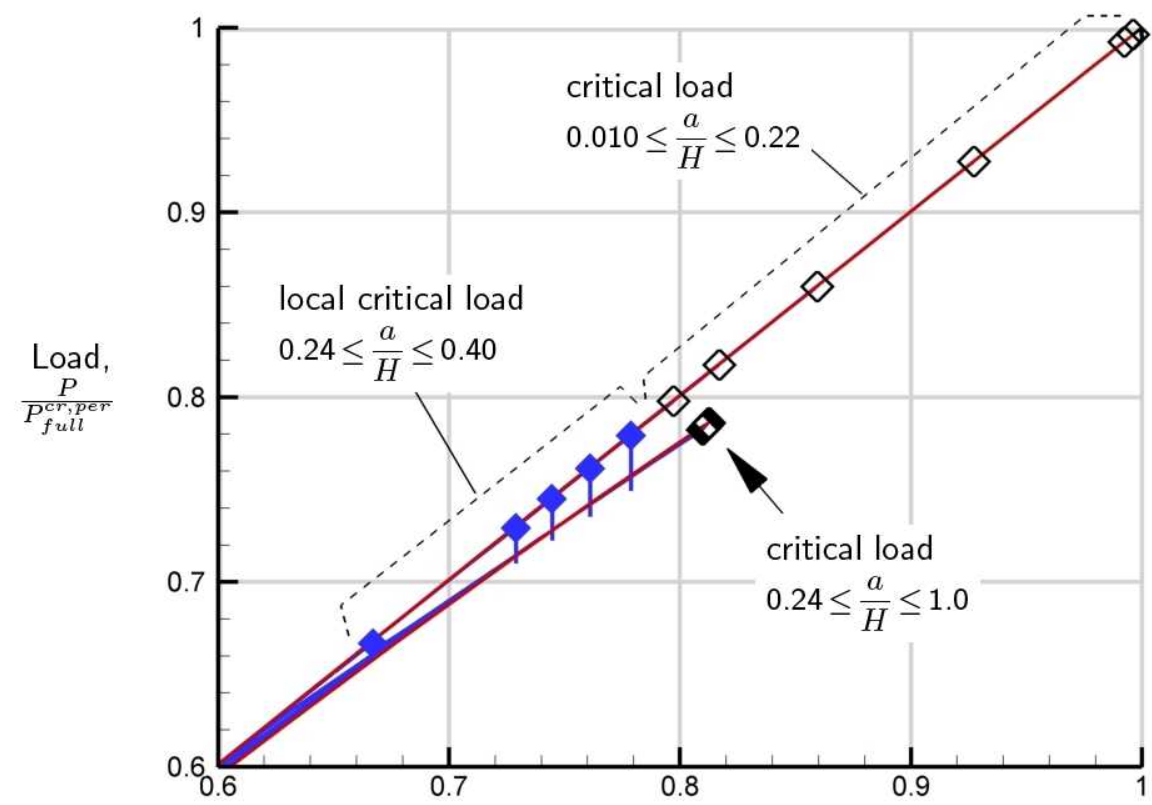

Axial End Displacement, $\frac{\delta}{\delta_{f u l l}^{\text {cr,per }}}$

(c) load - end displacement responses for different imperfection amplitudes

Figure 14. Effect of single dimple imperfection amplitude on full-scale cylinder response 


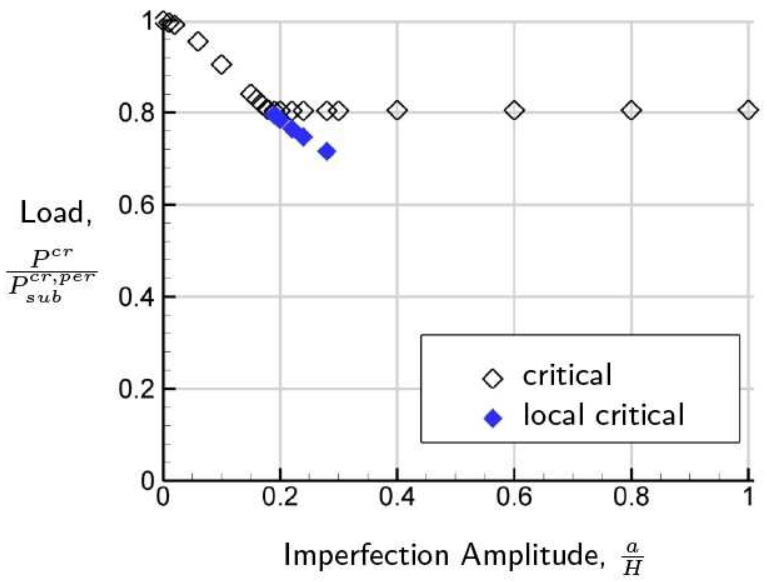

(a) critical load - imperfection amplitude relationship

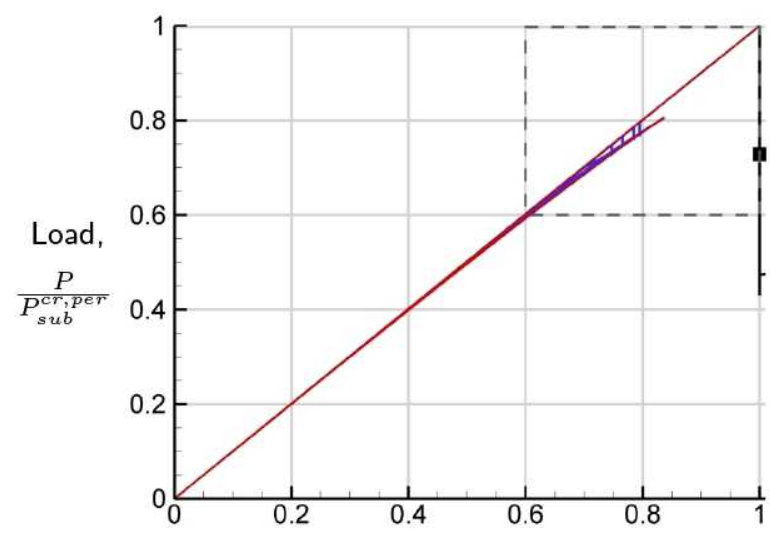

Axial End Displacement, $\frac{\delta}{\delta_{\text {sub }}^{c r, p e r}}$

(b) load - end displacement responses for different imperfection amplitudes

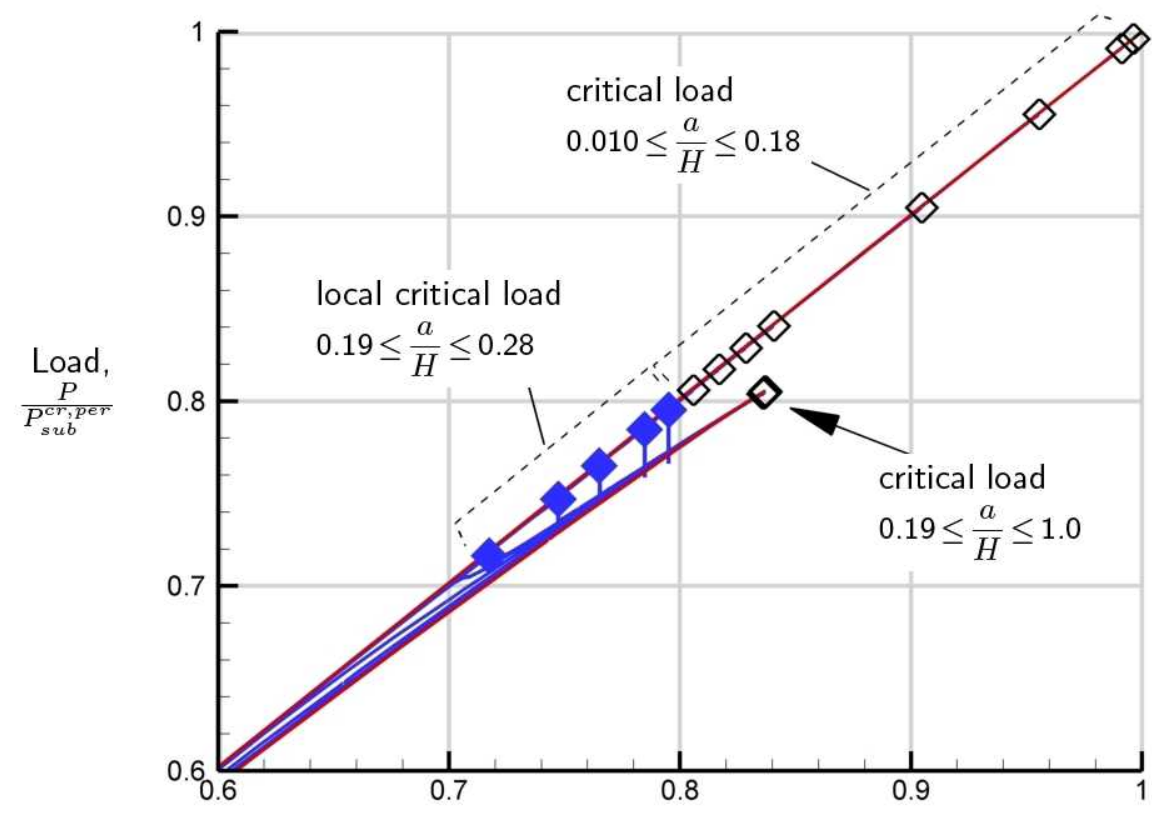

Axial End Displacement, $\frac{\delta}{\delta_{\text {sub }}^{\text {cr,per }}}$

(c) load - end displacement responses for different imperfection amplitudes

Figure 15. Effect of single dimple imperfection amplitude on sub-scale cylinder response 


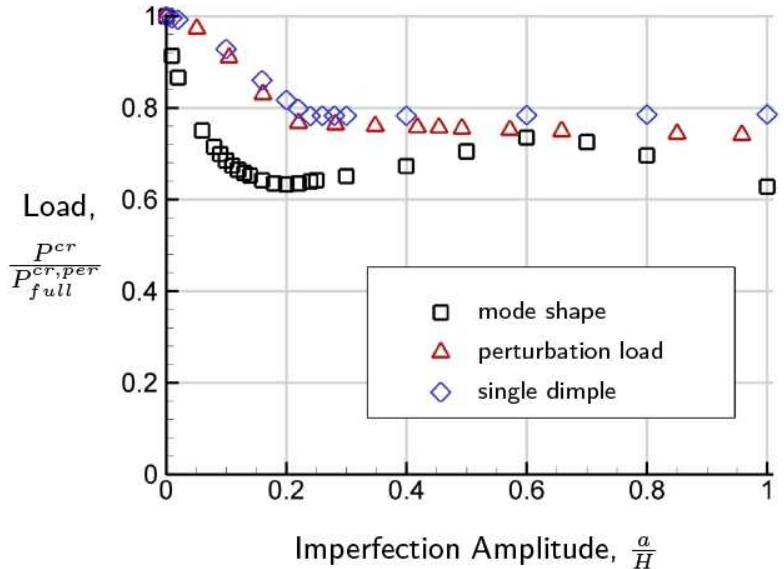

(a) full-scale

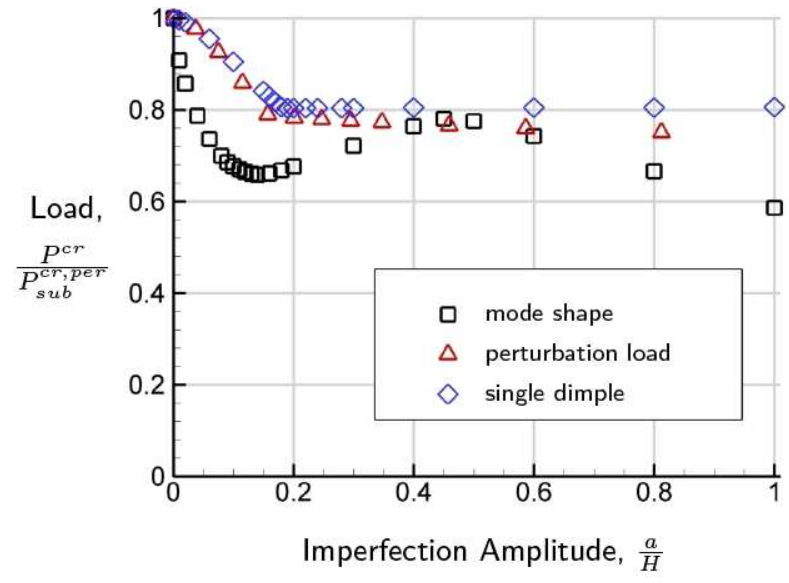

(b) sub-scale

Figure 16. Effect of imperfection type and amplitude on critical axial load of cylinder 